\title{
Photoproduction of electron-positron pairs on the proton in the resonance region
}

\author{
A. Yu. Korchin ${ }^{a, b}$ and O. Scholten ${ }^{a}$ \\ ${ }^{a}$ Kernfysisch Versneller Instituut, University of Groningen, 9747 AA Groningen, \\ The Netherlands \\ ${ }^{b}$ National Science Center 'Kharkov Institute of Physics and Technology', 310108 Kharkov, \\ Ukraine
}

\begin{abstract}
Production of lepton pairs in the $\gamma p \rightarrow e^{-} e^{+} p$ reaction is studied at photon energies up to $1 \mathrm{GeV}$. We show that even if lepton charge is not measured there are extensive kinematical regimes where the nuclear process dominates over the Bethe-Heitler contribution. The decomposition is performed of the unpolarized cross section for the virtual Compton scattering in terms of response functions. These are expressed in terms of a polarization density matrix of the virtual photon and are shown to be sensitive to the properties of baryon resonances in the first and the second resonance regions. In the analysis a unitary K-matrix model is used, based on an effective Lagrangian including nucleon, mesons, and baryon resonances with masses up to $1.7 \mathrm{GeV}$. Results of the model are compared with data for real-photon Compton scattering and predictions are given for observables in photoproduction of lepton pairs.
\end{abstract}

1996 PACS numbers: 13.30.-a, 13.40.-f, 13.40.Gp, 13.40.Hq, 13.60.Fz

Key Words Virtual Compton scattering; Real Compton scattering; Baryon resonances; K-matrix approach

\section{INTRODUCTION}

Compton scattering (CS) on the proton with real and virtual photons is a fundamental process which provides information on the internal structure of the proton and its excited states. Virtual CS where the initial photon is space-like, i.e. the reaction $e^{-} p \rightarrow e^{-} p \gamma$, has recently attracted considerable attention. Below the pion production threshold it allows, via an interference of the Bethe-Heitler $(\mathrm{BH})$ process with the proton virtual $\mathrm{CS}$, to measure the generalized polarizabilities of the proton. For a review we refer to [1].2].

Virtual CS in the time-like region, the reaction $\gamma p \rightarrow e^{-} e^{+} p$, is of interest for complementarity reasons. The process is sensitive to the nucleon electromagnetic (e.m.) form factors in the region $0<k^{2}<4 m^{2}$ (where $m$ is the nucleon mass, $k$ is the photon momentum), which are not accessible in elastic electron-proton scattering or $e^{+} e^{-}$annihilation to $p \bar{p}$. This possibility was explored in Ref. [3]. In the present paper the main interest is to 
investigate dependence of the polarization density matrix of the virtual photon on various contributions to the nuclear matrix element.

In certain parts of phase space the cross section is dominated by the BH process. Only when lepton charges are measured this can be turned to an advantage by measuring an $e^{+} e^{-}$asymmetry which is directly proportional to the virtual CS - BH interference. This was suggested long ago [4] and more recently elaborated in [5] 7].

In this paper we study the situation where the electron and positron in the $\gamma p \rightarrow e^{-} e^{+} p$ reaction are not distinguished which is the case for experiments which are being done at MAMI (Mainz) [8]. Under such conditions, because of the different charge-conjugation parity of virtual $\mathrm{CS}$ and $\mathrm{BH}$ amplitudes [9], the above interference vanishes and one is left with the incoherent sum of virtual CS and BH contributions. We show that there are large kinematical regimes where the more interesting virtual CS on the proton is dominant, mainly at backward angles for the virtual photon. In these conditions the (unpolarized) $\gamma N \rightarrow e^{-} e^{+} N$ cross section can be decomposed in terms of response functions (RF)s, like is usually done for exclusive electron scattering on nuclei (see, e.g. [10]). In addition to the transverse RF, which can also be measured in unpolarized CS with real photons, there are three more RFs for dilepton production. These are directly related to polarization properties of the virtual photon. To make this link more transparent we apply to the $\gamma N \rightarrow \gamma^{*} N$ reaction the density matrix formalism developed [11] for the photoproduction of vector mesons on the proton.

Differential cross sections and RFs for the $\gamma p \rightarrow e^{-} e^{+} p$ reaction are calculated in the unitary K-matrix model previously developed [12,13 for pion-nucleon scattering, pion photoproduction and real-photon CS. Before applying the model to virtual CS we compare its predictions to data for real CS in the energy region up to $1 \mathrm{GeV}$.

In the calculation of virtual CS we concentrate on the 1st and the 2nd resonance regions which are clearly seen in the cross section for real CS. The cross section and elements of the polarization density matrix $\rho_{\lambda \lambda^{\prime}}$ are calculated for two energies corresponding to excitation of the $P_{33}(1232)$ and the $D_{13}(1520)$ resonances. We study effects of different reaction mechanisms on these observables. In particular, for the $\Delta$ region it is of interest to investigate the effect of t-channel exchange of the $\sigma$ meson.

At photon energies of about $700 \mathrm{MeV}$ photon invariant masses up to $\approx 540 \mathrm{MeV}$ become accessible. In this energy regime several contributions are important, in particular, the $D_{13}(1520)$ and $S_{11}(1535)$ resonances, and $\sigma$ exchange. Due to their different influence on the density matrix elements $\rho_{\lambda \lambda^{\prime}}$ these contributions can be distinguished in an experiment provided RFs are extracted. Cross section and other observables are also sensitive to the $G_{3}\left(k^{2}\right)$ coupling in the $\gamma N D_{13}(1520)$ vertex which is specific for virtual photon and does not contribute to real CS.

The paper is organized as follows. In sect. II we specify variables and give the expression for the exclusive cross section. The decomposition of the virtual CS cross section in terms of RFs is presented. Relations between RFs and polarization density matrix elements are established and the limit of "almost" real photons is considered. We briefly discuss ingredients of the dynamical model used and compare calculated cross sections and polarization observables with data for real CS. In sect. III kinematical regimes are investigated where the $\mathrm{BH}$ contribution can be neglected compared to virtual CS. Results for response $W_{S}=W_{T}+W_{L}$ and elements of the photon density matrix are presented. Conclusions are given in sect. IV. 
In Appendix A expressions for RFs for virtual CS and polarization observables for real CS in terms of helicity amplitudes are collected.

\section{FORMALISM}

\section{A. Cross section for $\gamma p \rightarrow e^{-} e^{+} p$ reaction}

If $q=\left(q_{0}=|\vec{q}|, \vec{q}\right)$ is the momentum of the incoming real photon and $k=\left(k_{0}, \vec{k}\right)=k_{-}+k_{+}$ the momentum of the outgoing virtual photon (see Fig. (1)), the fully exclusive cross section in the c.m. frame can be written as 1

$$
\frac{d \sigma\left(e^{-} e^{+}\right)}{d M_{\gamma} d \Omega_{\gamma} d \Omega_{l}}=\mathcal{K}_{e^{-} e^{+}}\left|A_{B H}+A_{V C S}\right|^{2}
$$

where $M_{\gamma}=\sqrt{k^{2}}$ is the photon invariant mass and $\mathcal{K}_{e^{-} e^{+}}$is the kinematical factor

$$
\mathcal{K}_{e^{-} e^{+}}=\frac{m^{2} m_{e}^{2}}{2(2 \pi)^{5} s} \frac{|\vec{k}|}{|\vec{q}|} \frac{4|\vec{l}|^{3}}{M_{\gamma} \beta^{2} k_{0}} \text {. }
$$

Here $m\left(m_{e}\right)$ is the proton (electron) mass, $\beta=\left(1-4 m_{e}^{2} / M_{\gamma}^{2}\right)^{1 / 2}$ is the $e^{-}\left(e^{+}\right)$velocity in the virtual-photon rest frame, and $s$ is the invariant energy squared. The average over initial and sum over final polarizations of all particles is implied in Eq. (1) as well as in the following.

To describe the $e^{-} e^{+}$pair the relative 4-momentum $l=\frac{1}{2}\left(k_{-}-k_{+}\right)$has been introduced [15]. The orientation of $\vec{l}$ is determined by the polar angle $\theta_{l}$ and the azimuthal (or out-of-plane) angle $\phi_{l}$ which are defined in the frame with $O Z$ axis along $\vec{k}$ and $O Y$ axis along $\vec{k} \times \vec{q}$. The magnitude of $\vec{l}$ is given by

$$
|\vec{l}|=\frac{M_{\gamma} \beta k_{0}}{2\left(M_{\gamma}^{2}+\vec{k}^{2} \sin ^{2} \theta_{l}\right)^{1 / 2}}
$$

with $k_{0}=\left(s-m^{2}+M_{\gamma}^{2}\right) / 2 \sqrt{s}$ and $\vec{k}^{2}=k_{0}^{2}-M_{\gamma}^{2}$. The solid angle differential $d \Omega_{l}$ stands for $d \cos \theta_{l} d \phi_{l}$, and $d \Omega_{\gamma}=d \cos \theta_{\gamma} d \phi_{\gamma}$, where $\theta_{\gamma}$ is the angle between vectors $\vec{k}$ and $\vec{q}$. The azimuthal angle $\phi_{\gamma}$ is superfluous and can be chosen zero. For more details about kinematics we refer to previous papers on $e^{-} e^{+}$production in capture reactions [15, 16] and $N N$ bremsstrahlung [17].

The Bethe-Heitler $\left(A_{B H}\right)$ and the virtual CS $\left(A_{V C S}\right)$ amplitudes are functions of five variables: $s, M_{\gamma}, \theta_{\gamma}, \theta_{l}$ and $\phi_{l}$. Before proceeding further one can exploit the different chargeconjugation properties of these amplitudes [9]. Interchanging variables describing $e^{-}$and $e^{+}$, which means $\vec{l} \rightarrow-\vec{l}$ and interchange $e^{-}$and $e^{+}$helicities, leads to the relations

\footnotetext{
${ }^{1}$ We follow notation of Ref. 14
} 


$$
\begin{aligned}
& \left|A_{B H}\left(\theta_{l}, \phi_{l}\right)\right|^{2}=\left|A_{B H}\left(\pi-\theta_{l}, \pi+\phi_{l}\right)\right|^{2}, \\
& \left|A_{V C S}\left(\theta_{l}, \phi_{l}\right)\right|^{2}=\left|A_{V C S}\left(\pi-\theta_{l}, \pi+\phi_{l}\right)\right|^{2}, \\
& A_{B H}^{*}\left(\theta_{l}, \phi_{l}\right) A_{V C S}\left(\theta_{l}, \phi_{l}\right)=-A_{B H}^{*}\left(\pi-\theta_{l}, \pi+\phi_{l}\right) A_{V C S}\left(\pi-\theta_{l}, \pi+\phi_{l}\right),
\end{aligned}
$$

where $\theta_{l}$ varies from $0^{\circ}$ to $180^{\circ}$ and only the dilepton angles are indicated. Therefore, if the electron and positron cannot be distinguished in an experiment, the cross section is the incoherent sum $d \bar{\sigma}\left(e^{-} e^{+}\right)=d \sigma\left(e^{-} e^{+}\right)+d \sigma\left(e^{+} e^{-}\right)$, which results in

$$
\frac{d \bar{\sigma}\left(e^{-} e^{+}\right)}{d M_{\gamma} d \Omega_{\gamma} d \Omega_{l}}=2 \mathcal{K}_{e^{-} e^{+}}\left(\left|A_{B H}\right|^{2}+\left|A_{V C S}\right|^{2}\right),
$$

where the interference term drops out.

Another interesting observable, the $e^{-} e^{+}$asymmetry, which is proportional to the virtual

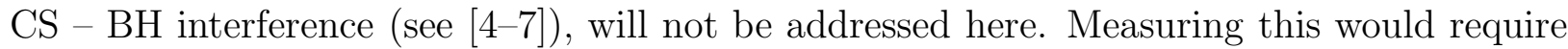
detection of the charges of the leptons.

The expression for $A_{B H}$ can be read off the diagrams g,h in Fig. (1). After some algebra it takes the form

$$
\begin{aligned}
& A_{B H}=\frac{e^{3}}{Q^{2}} \bar{u}\left(k_{-}\right)[\left.\gamma_{\mu}\left(\frac{k_{+}^{\nu}}{k_{+} \cdot q}-\frac{k_{-}^{\nu}}{k_{-} \cdot q}\right)+\frac{\gamma^{\nu} \phi \gamma_{\mu}}{2 k_{-} \cdot q}-\frac{\gamma_{\mu} \phi \gamma^{\nu}}{2 k_{+} \cdot q}\right] v\left(k_{+}\right) \\
& \times \bar{u}\left(p^{\prime}, \Lambda^{\prime}\right)\left[F_{1}\left(Q^{2}\right) \gamma^{\mu}+i \frac{\sigma^{\mu \rho} Q_{\rho}}{2 m} F_{2}\left(Q^{2}\right)\right] u(p, \Lambda) \epsilon_{\nu}(\lambda),
\end{aligned}
$$

where $u(p, \Lambda)\left(\bar{u}\left(p^{\prime}, \Lambda^{\prime}\right)\right)$ is the spinor of the initial (final) nucleon with momentum $p\left(p^{\prime}\right)$ and helicity $\Lambda\left(\Lambda^{\prime}\right)$. In Eq. (6) $e$ is the proton charge, $\bar{u}\left(k_{-}\right)$and $v\left(k_{+}\right)$are the lepton spinors (omitting spin indices), and $\epsilon(\lambda)$ is the photon polarization vector for helicity $\lambda$. The nucleon e.m. form factors $F_{1,2}\left(Q^{2}\right)$ depend on the momentum $Q=p^{\prime}-p$.

The virtual CS matrix element is

$$
A_{V C S}=\frac{e^{2}}{M_{\gamma}^{2}} \bar{u}\left(k_{-}\right) \gamma_{\mu} v\left(k_{+}\right) J^{\mu},
$$

where the e.m. current $J^{\mu}$ is expressed through the CS tensor $M^{\mu \nu}$

$$
J^{\mu}=e \bar{u}\left(p^{\prime}, \Lambda^{\prime}\right) M^{\mu \nu} u(p, \Lambda) \epsilon_{\nu}(\lambda) .
$$

Some of the ingredients in the calculation of $M^{\mu \nu}$ are discussed in sect. IIT.

Note that the real CS matrix element $\left(A_{R C S}\right)$ can be written in this notation as

$$
A_{R C S}=e^{2} \epsilon_{\mu}^{\prime *}\left(\lambda^{\prime}\right) \bar{u}\left(p^{\prime}, \Lambda^{\prime}\right) M^{\mu \nu} u(p, \Lambda) \epsilon_{\nu}(\lambda)
$$

with $\epsilon^{\prime}\left(\lambda^{\prime}\right)$ being the transverse polarization vector of the final photon which is real in this case.

\section{B. Response functions and polarization density matrix}

In kinematics where virtual CS is the dominant process it is of interest to decompose the cross section in terms of response functions (RF)s, similar to what is common practice 
in the space-like region. We will not go in much detail because the derivation is similar to $e^{-} e^{+}$production in other reactions 15 17.

After summing over lepton polarizations and making use of gauge invariance $k \cdot J=0$ one obtains in the c.m. frame

$$
\begin{aligned}
& \frac{d \bar{\sigma}\left(e^{-} e^{+}\right)}{d M_{\gamma} d \Omega_{\gamma} d \Omega_{l}}=\frac{\alpha^{2} m^{2}|\vec{l}|^{3}}{2 \pi^{3} s M_{\gamma}^{3} \beta^{2} k_{0}} \frac{|\vec{k}|}{|\vec{q}|} 2 \mathcal{S}, \\
& \mathcal{S}=W_{T} x_{T}+W_{L} x_{L}+W_{T T} x_{T T} \cos 2 \phi_{l}+W_{L T} x_{L T} \cos \phi_{l},
\end{aligned}
$$

where $\alpha$ is the fine-structure constant, $W_{i} \equiv W_{i}\left(s, \theta_{\gamma}, M_{\gamma}\right)$ are RFs for $i=T, L, T T$ and $L T$, and the factor 2 in front of $\mathcal{S}$ comes from the definition Eq. (5). Dependence on the polar angle $\theta_{l}$ is contained in the factors $x_{i}$

$$
\begin{array}{rlrl}
x_{T} & =1-2 \frac{\vec{l}^{2}}{M_{\gamma}^{2}} \sin ^{2} \theta_{l}, & x_{L} & =1-4 \frac{\vec{l}^{2}}{k_{0}^{2}} \cos ^{2} \theta_{l}, \\
x_{T T} & =2 \frac{\vec{l}^{2}}{M_{\gamma}^{2}} \sin ^{2} \theta_{l}, & x_{L T}=\sqrt{2} \frac{\vec{l}^{2}}{M_{\gamma} k_{0}} \sin 2 \theta_{l},
\end{array}
$$

where $|\vec{l}|$, which also depends on $\theta_{l}$, is defined in Eq. (3).

RFs are defined in terms of the space components $J^{i}$ of the e.m. current as [16]

$$
\begin{array}{ll}
W_{T}=\frac{1}{4} \sum_{\text {polar. }}\left|J_{x}\right|^{2}+\left|J_{y}\right|^{2}, & W_{L}=\frac{1}{4} \frac{k^{2}}{k_{0}^{2}} \sum_{\text {polar. }}\left|J_{z}\right|^{2}, \\
W_{T T}=\frac{1}{4} \sum_{\text {polar. }}\left|J_{y}\right|^{2}-\left|J_{x}\right|^{2}, & W_{L T}=-\frac{1}{4} \frac{\sqrt{k^{2}}}{k_{0}} \sum_{\text {polar. }} 2 \sqrt{2} \Re\left(J_{z}^{*} J_{x}\right),
\end{array}
$$

where the sum runs over the proton and initial photon polarizations.

It is interesting to note that certain combinations of the e.m. current vanish due to reflection symmetry $(O Y \rightarrow-O Y)$ with respect to the scattering plane $O X Z$ :

$$
\sum_{\text {polar. }} \Re\left(J_{x}^{*} J_{y}\right)=\sum_{\text {polar. }} \Im\left(J_{x}^{*} J_{y}\right)=\sum_{\text {polar. }} \Re\left(J_{z}^{*} J_{y}\right)=\sum_{\text {polar. }} \Im\left(J_{z}^{*} J_{y}\right)=0 .
$$

These constraints are specific for a two-body final state such as $N+\gamma^{*}$ and may be useful for checking consistency of a model.

Experimental separation of RFs can be performed using the different dependencies of the kinematical factors in Eq. (11) on the dilepton angles. In Refs. 18 an example of such a separation for the case of $N N$ virtual bremsstrahlung is discussed.

To obtain some further insight we relate the above RFs to elements of the polarization density matrix $\hat{\rho}$ of the virtual photon. The formalism of the polarization density matrix has been used before in production of vector mesons $\rho, \omega$ (see Refs. [19,11,20,21]). One introduces the $3 \times 3$ density matrix for a spin-1 particle [1]

$$
\hat{\rho}=\left(\begin{array}{ccc}
\rho_{11} & \rho_{10} & \rho_{1-1} \\
\rho_{10}^{*} & \rho_{00} & -\rho_{10}^{*} \\
\rho_{1-1} & -\rho_{10} & \rho_{11}
\end{array}\right),
$$


in the helicity basis $\lambda, \lambda^{\prime}=0, \pm 1$. It satisfies the conditions of hermiticity $\rho_{\lambda \lambda^{\prime}}=\rho_{\lambda^{\prime} \lambda}^{*}$, parity conservation $\rho_{\lambda \lambda^{\prime}}=(-1)^{\lambda-\lambda^{\prime}} \rho_{-\lambda-\lambda^{\prime}}$, and has a unit trace $\left(\rho_{11}=\frac{1}{2}\left(1-\rho_{00}\right)\right)$. $\rho_{00}$ and $\rho_{1-1}$ are thus real quantities while the element $\rho_{10}$ is, in general, complex.

The form Eq. (15) is valid only for a reaction with a two-body final state (like $N+\gamma^{*}$ ), in which the initial hadron and photon are not polarized and the polarization of the final hadron is not measured. If the initial photon in $\gamma N \rightarrow \gamma^{*} N$ is (fully or partially) polarized the general density matrix is more complicated [11].

From the density matrix and the $\gamma e^{-} e^{+}$vertex, which is well-known from QED, one can obtain the angular distribution $F\left(\bar{\theta}_{l}, \bar{\phi}_{l}\right)$ of $\gamma^{*}$ decay into $e^{-} e^{+}$. In the virtual-photon rest frame it takes the form (see Ref. [22], ch. 4-13 2 )

$$
\begin{aligned}
F\left(\bar{\theta}_{l}, \bar{\phi}_{l}\right)=\frac{1}{4 \pi}\left\{1-\frac{\beta^{2}}{3-\beta^{2}}[\right. & \frac{1}{2}\left(3 \rho_{00}-1\right)\left(3 \cos ^{2} \bar{\theta}_{l}-1\right) \\
& \left.\left.-3 \rho_{1-1} \sin ^{2} \bar{\theta}_{l} \cos 2 \bar{\phi}_{l}-3 \sqrt{2} \Re \rho_{10} \sin 2 \bar{\theta}_{l} \cos \bar{\phi}_{l}\right]\right\},
\end{aligned}
$$

where $\beta$ is defined after Eq. (2) and $\left(\bar{\theta}_{l}, \bar{\phi}_{l}\right)$ denote the dilepton angles.

Expression Eq. (11) in terms of RFs, boosted to the $\gamma^{*}$ rest frame, can be cast in the form (details on the angle transformation can be found in Refs. [15, 17])

$$
\begin{aligned}
\mathcal{S} & =W_{T}\left(1-\frac{1}{2} \beta^{2} \sin ^{2} \bar{\theta}_{l}\right)+W_{L}\left(1-\beta^{2} \cos ^{2} \bar{\theta}_{l}\right)+W_{T T} \frac{1}{2} \beta^{2} \sin ^{2} \bar{\theta}_{l} \cos 2 \bar{\phi}_{l} \\
& +W_{L T} \frac{1}{2 \sqrt{2}} \beta^{2} \sin 2 \bar{\theta}_{l} \cos \bar{\phi}_{l} \\
& =\left(1-\frac{\beta^{2}}{3}\right) W_{S}\left\{1-\frac{\beta^{2}}{3-\beta^{2}}\left[\frac{2 W_{L}-W_{T}}{2 W_{S}}\left(3 \cos ^{2} \bar{\theta}_{l}-1\right)-\frac{3 W_{T T}}{2 W_{S}} \sin ^{2} \bar{\theta}_{l} \cos 2 \bar{\phi}_{l}\right.\right. \\
& \left.\left.-\frac{3 W_{L T}}{2 \sqrt{2} W_{S}} \sin 2 \bar{\theta}_{l} \cos \bar{\phi}_{l}\right]\right\},
\end{aligned}
$$

where $W_{S} \equiv W_{T}+W_{L}$. It is seen that the angle dependent part of $\mathcal{S}$ has the same structure as $F\left(\bar{\theta}_{l}, \bar{\phi}_{l}\right)$ in Eq. (16). Comparing terms with the same angle factors one obtains

$$
\rho_{00}=\frac{W_{L}}{W_{S}}, \quad \rho_{1-1}=\frac{W_{T T}}{2 W_{S}}, \quad \Re \rho_{10}=\frac{W_{L T}}{4 W_{S}} .
$$

Note that the imaginary part of $\rho_{10}$ does not enter in the $\gamma^{*} \rightarrow e^{-} e^{+}$angular distribution (cf. [11] for $\gamma N \rightarrow \rho N \rightarrow \pi \pi N)$. For completeness we give an expression for $\Im \rho_{10}$ through an additional (independent) function $\tilde{W}_{L T}$ :

$$
\Im \rho_{10}=\frac{\tilde{W}_{L T}}{4 W_{S}}, \quad \quad \tilde{W}_{L T}=-\frac{1}{4} \frac{\sqrt{k^{2}}}{k_{0}} \sum_{\text {polar. }} 2 \sqrt{2} \Im\left(J_{z}^{*} J_{x}\right) .
$$

$W_{L T}$ and $\tilde{W}_{L T}$ can be regarded as the real and the imaginary parts of one complex function. Thus only three (out of four independent) components of the photon-polarization density

\footnotetext{
${ }^{2}$ There is a misprint in Eq.(13.14) of this reference: in front of $a_{1}$ there should be an additional factor of $1 / 2$
} 
matrix in addition to the cross section can be determined from measuring $W_{T}, W_{L}, W_{T T}$, and $W_{L T}$ in the $\gamma N \rightarrow e^{-} e^{+} N$ reaction with unpolarized leptons.

Since $\left|W_{T T}\right| \leq W_{T} \leq W_{S}$ (see definitions in Eqs. (13)), the following inequalities are in order

$$
0 \leq \rho_{00} \leq 1, \quad\left|\rho_{1-1}\right| \leq \frac{1}{2}\left(1-\rho_{00}\right) \leq \frac{1}{2} .
$$

The upper bound for $\rho_{10}$ was obtained in Ref. [19] using the Schwartz inequality

$$
\left|\rho_{10}\right| \leq \frac{1}{2} \sqrt{\rho_{00}\left(1-\rho_{00}-2 \rho_{1-1}\right)} \leq \frac{1}{\sqrt{2}} \sqrt{\rho_{00}\left(1-\rho_{00}\right)} \leq \frac{1}{2 \sqrt{2}} .
$$

Additional constraints have recently been derived in [21].

Sometimes (see [23, 20]) the density matrix for the spin-1 particle is expressed in terms of tensor and vector polarizations

$$
\hat{\rho}=\frac{1}{3}\left(\begin{array}{ccc}
1+\frac{1}{\sqrt{2}} t_{20} & \sqrt{\frac{3}{2}} t_{11}-\sqrt{\frac{3}{2}} t_{21} & \sqrt{3} t_{22} \\
-\sqrt{\frac{3}{2}} t_{11}-\sqrt{\frac{3}{2}} t_{21} & 1-\sqrt{2} t_{20} & \sqrt{\frac{3}{2}} t_{11}+\sqrt{\frac{3}{2}} t_{21} \\
\sqrt{3} t_{22} & -\sqrt{\frac{3}{2}} t_{11}+\sqrt{\frac{3}{2}} t_{21} & 1+\frac{1}{\sqrt{2}} t_{20}
\end{array}\right),
$$

where the tensor polarizations $t_{20}, t_{21}$ and $t_{22}$ are real, and $t_{11}$ is purely imaginary and related to the vector polarization $p_{y}$ via $t_{11}=-i \frac{\sqrt{3}}{2} p_{y}$. Relations between these polarizations and elements $\rho_{\lambda \lambda^{\prime}}$ follow from Eq. (15) and Eq. (22),

$$
\rho_{00}=\frac{1}{3}\left(1-\sqrt{2} t_{20}\right), \quad \rho_{1-1}=\frac{1}{\sqrt{3}} t_{22}, \quad \Re \rho_{10}=-\frac{1}{\sqrt{6}} t_{21}, \quad \Im \rho_{10}=-\frac{i}{\sqrt{6}} t_{11} .
$$

Using Eqs. (18) and Eqs. (23) one can express the polarizations in terms of RFs

$$
\begin{aligned}
t_{20} & =\frac{1}{\sqrt{2}} \frac{W_{T}-2 W_{L}}{W_{S}}, & t_{22} & =\frac{\sqrt{3}}{2} \frac{W_{T T}}{W_{S}}, \\
t_{21} & =-\frac{\sqrt{3}}{2 \sqrt{2}} \frac{W_{L T}}{W_{S}}, & t_{11} & =i \frac{\sqrt{3}}{2 \sqrt{2}} \frac{\tilde{W}_{L T}}{W_{S}} .
\end{aligned}
$$

As a last point it is interesting to consider the limiting case of small invariant masses $M_{\gamma}$ close to the threshold value $M_{\gamma}^{t h}=2 m_{e}$. In this case the longitudinal component of the e.m. current becomes negligibly small, and elements $\rho_{00}$ and $\rho_{10}$ vanish. As follows from Eqs. (24), $t_{20} \rightarrow 1 / \sqrt{2}, t_{21}, t_{11} \rightarrow 0$. In this limit Eq. (15) reduces to

$$
\hat{\rho}=\frac{1}{2}\left(\begin{array}{ccc}
1 & 0 & \frac{W_{T T}}{W_{T}} \\
0 & 0 & 0 \\
\frac{W_{T T}}{W_{T}} & 0 & 1
\end{array}\right) .
$$

From a physical point of view this limit is close to real CS. A real photon is, in general, described by the $2 \times 2$ density matrix in the helicity basis [11]

$$
\hat{\rho}_{\gamma}=\frac{1}{2}\left(\begin{array}{cc}
1 \pm I_{C} & -I_{L} \exp (-2 i \psi) \\
-I_{L} \exp (2 i \psi) & 1 \mp I_{C}
\end{array}\right)
$$


where $I_{L}\left(I_{C}\right)$ is the degree of linear (circular) polarization, $\psi$ is the angle between the direction of linear polarization and the $O X$ axis, and $+(-)$ stands for the right (left) circular polarization. Comparing Eq. (26) with Eq. (25) (dropping there the superfluous line and column with elements equal to zero) one finds

$$
\begin{aligned}
& I_{C}=0, \quad I_{L}=\frac{\left|W_{T T}\right|}{W_{T}}<1, \\
& \psi=\left\{\begin{array}{lll}
0, & \text { if } & \operatorname{sign}\left(W_{T T}\right)=-1 \\
\frac{\pi}{2}, & \text { if } & \operatorname{sign}\left(W_{T T}\right)=+1
\end{array}\right.
\end{aligned}
$$

Thus the "quasi-real" photon has no circular polarization, and may have a linear polarization along the $O X$ axis (in the scattering plane), or along the $O Y$ axis (perpendicular to the scattering plane) depending on the sign of the interference $W_{T T}$. Note that this is a particular feature of any reaction with unpolarized particles in coplanar kinematics and can be understood based on reflection symmetry with respect to the scattering plane.

The photon asymmetry [24], as can be measured in real CS with a linearly-polarized photon beam, is related to $W_{T}^{r}$ and $W_{T T}^{r}$ (the superscript "r" denotes taking the real CS limit). If there are no bound states in the energy regime of interest, the RFs for $e^{-} e^{+}$ production behave smoothly as functions of $M_{\gamma}\left[\right.$ and the threshold values $W_{T, T T}$ at $M_{\gamma}=$ $2 m_{e}$ can be used to calculate $W_{T, T T}^{r}$. Making use of Eq. (26) and time-reversal invariance one obtains for the real CS cross section

$$
\frac{d \sigma(\gamma)}{d \Omega_{\gamma}}=\frac{\alpha m^{2}}{4 \pi s}\left(W_{T}^{r}-W_{T T}^{r} I_{L} \cos 2 \psi\right),
$$

where the angle $\psi$ specifies the direction of the incoming photon polarization and all other particles are not polarized. The cross section for unpolarized photons is expressed via $W_{T}^{r}$, and the asymmetry is proportional to the interference $W_{T T}^{r}$ :

$$
\Sigma_{\gamma}=\frac{d \sigma_{\perp}(\gamma)-d \sigma_{\|}(\gamma)}{d \sigma_{\perp}(\gamma)+d \sigma_{\|}(\gamma)}=\frac{W_{T T}^{r}}{W_{T}^{r}} .
$$

Calculations of the cross section and RFs for virtual and real CS are performed in the helicity formalism (see Appendix A where details are presented).

It is of interest to make a link between the representation in Eq. (11) and the longitudinaltransverse $(\mathrm{L}-\mathrm{T})$ decomposition established for electron scattering on nuclei. At invariant masses $M_{\gamma}$ considerably larger than $2 m_{e}$ one can rewrite Eq. (11) as

$$
\begin{aligned}
\mathcal{S}=2 x_{T}\left[\left(\frac{1}{2} W_{T}\right)-\varepsilon_{+}\left(-W_{L}\right)\right. & -\varepsilon_{+} \cos 2 \phi_{l}\left(-\frac{1}{2} W_{T T}\right) \\
& \left.+\eta \sqrt{2 \varepsilon_{+}\left(1-\varepsilon_{+}\right)} \cos \phi_{l}\left(\frac{1}{2 \sqrt{2}} W_{L T}\right)\right],
\end{aligned}
$$

\footnotetext{
${ }^{3}$ In principle, kinematical conditions exist when the electron interacts strongly with the proton via the Coulomb attraction and may be captured in a hydrogen atom. However, this happens in a tiny region of the phase space and is neglected in the present model. Likewise the Coulomb interaction between the $e^{-}$and the $e^{+}$and possible formation of positronium is not considered
} 
where $\eta=\operatorname{sign}\left(\sin 2 \theta_{l}\right)$, and $\varepsilon_{+}=\left(1-x_{T}\right) / x_{T}$ which satisfies the conditions $0<\varepsilon_{+}<1$. Expression Eq. (30) is similar to the $L-T$ decomposition used in electron scattering (see e.g., [10,25]) To obtain exactly the same form one can make in Eq. (30) the formal substitutions $k^{2} \rightarrow-\left|k^{2}\right|$ and $\varepsilon_{+} \rightarrow-\varepsilon_{-}$. This results in

$$
\mathcal{S}=2 x_{T}\left[R_{T}+\varepsilon_{-} R_{L}+\varepsilon_{-} \cos 2 \phi_{l} R_{T T}+\eta \sqrt{2 \varepsilon_{-}\left(1+\varepsilon_{-}\right)} \cos \phi_{l} R_{L T}\right],
$$

where RFs for the electron scattering, denoted by $R_{i}$, are related to $W_{i}$ via

$$
R_{T}=\frac{1}{2} W_{T}, \quad R_{L}=-W_{L}, \quad R_{T T}=-\frac{1}{2} W_{T T}, \quad R_{L T}=-\frac{i}{2 \sqrt{2}} W_{L T} .
$$

Comparison of Eq. (31) with the $L-T$ decomposition in [25] (Eqs. $(22,23))$ suggests that $\varepsilon_{-}$ has a meaning of degree of transverse polarization of the space-like photon. The interference $R_{L T}$ is of course a real function in this case since the factor $i$ merely accounts for $\sqrt{k^{2}} \rightarrow$ $i \sqrt{\left|k^{2}\right|}$ in the definition of Eqs. (13).

\section{Description of the model and comparison with data for real Compton scattering}

As mentioned in sect. 1 we employ the K-matrix model which is unitary in the channel space $\pi N \oplus \gamma N$. The tree-level K-matrix includes the four-star baryon resonances: $\mathrm{P}_{11}(\mathrm{~N} 1440), \mathrm{D}_{13}(\mathrm{~N} 1520), \mathrm{S}_{11}(\mathrm{~N} 1535), \mathrm{S}_{11}(\mathrm{~N} 1650), \mathrm{P}_{33}(\Delta 1232), \mathrm{S}_{31}(\Delta 1620)$ and $\mathrm{D}_{33}(\Delta 1700)$ in the s- and u-type diagrams. The t-channel contributions come from the exchange of $\sigma$ and $\rho$ mesons (for $\pi N$ scattering), $\pi, \rho$, and $\omega$ mesons (for pion photoproduction), and $\pi^{0}$, $\eta$ and $\sigma$ mesons (for CS). The amplitude for CS is shown in Fig. (1), diagrams a - f. For more details about the model we refer to Ref. [13]. In order to calculate the virtual CS the longitudinally polarized photons have also been taken into account.

In this paper we concentrate on the $P_{33}(1232)$ and the $D_{13}(1520)$ resonance regions. The $\gamma N R$ vertex for the e.m. couplings of the spin-3/2 resonances is chosen as follows [26 29]

$V_{N \gamma \rightarrow R^{\alpha}}=\frac{i e}{2 m}\left[G_{1}\left(k^{2}\right) \theta_{\alpha \beta}\left(z_{1}\right) \gamma_{\delta}-\frac{G_{2}\left(k^{2}\right)}{2 m} \theta_{\alpha \beta}\left(z_{2}\right) p_{\delta}-\frac{G_{3}\left(k^{2}\right)}{2 m} \theta_{\alpha \beta}\left(z_{3}\right) k_{\delta}\right] \Gamma\left(k^{\beta} \epsilon^{\delta}-k^{\delta} \epsilon^{\beta}\right)$,

and $V_{R^{\alpha} \rightarrow N \gamma}=-\gamma_{0} V_{N \gamma \rightarrow R^{\alpha}}^{\dagger} \gamma_{0}$. In Eq. (33) $p(k)$ is the nucleon (photon) momentum and $\epsilon$ is the photon polarization vector. For isospin- $1 / 2$ resonances $G_{i}\left(k^{2}\right)=\left[g_{i}^{(p)}\left(1+\tau_{3}\right) / 2+\right.$ $\left.g_{i}^{(n)}\left(1-\tau_{3}\right) / 2\right] F_{V M D}\left(k^{2}\right)$, while for isospin-3/2 case $G_{i}\left(k^{2}\right)=g_{i} T_{3} F_{V M D}\left(k^{2}\right)$, where $T_{3}$ is $1 / 2 \leftrightarrow 3 / 2$ isospin-transition operator and $F_{V M D}\left(k^{2}\right)$ is the form factor in the extended VMD model [30]. Note that the $G_{3}\left(k^{2}\right)$ term contributes only for virtual photons. Further, $\Gamma=\left(\gamma_{5}, 1\right)$ for the resonances $\left(3 / 2^{+}, 3 / 2^{-}\right)$, and the tensor $\theta_{\alpha \beta}\left(z_{i}\right)=g_{\alpha \beta}+a_{i} \gamma_{\alpha} \gamma_{\beta}$ with $a_{i} \equiv-\left(1 / 2+z_{i}\right)$ contains the off-shell parameter $z_{i}$ [26,27], where $i=1,2,3$. In the present work we show calculations for two parameter sets "A" and "B": A) $a_{i}=0$ for all spin-3 $/ 2$ resonances, B) $a_{1}=0.12, a_{2}=0.5, a_{3}=0$ for the $D_{13}(1520)$, and $a_{i}=0$ for other resonances. The fitted values of the couplings $\left(g_{1}, g_{2}\right)$ are: $(4.9,5.27)$ for the $P_{33}(1232)$, $(7.25,7.9)$ for the $D_{13}(1520)$ (on the proton), and $(1.74,4.75)$ for the $D_{33}(1700)$.

The cross section for real CS on the proton and polarization observables are presented in Fig. (2) for photon energies up to $1 \mathrm{GeV}$. The cross section in the $\Delta$-resonance region 
agrees with the data as well as with other calculations which are based on dispersion relations 31, 33, 34, and an effective Lagrangian [35]. For comparison we show results of the calculations from Ref. [31] (dotted lines) as they extend to high energies. In Ref. [31] the imaginary part of the Compton amplitude is calculated directly from pion-photoproduction data and the real part is calculated using fixed- $t$ unsubtracted dispersion relations. Our model and 31 give the same results for the photon asymmetry $\Sigma_{\gamma}$ at the $\Delta$ resonance, however they predict a different slope. Similar disagreement was noticed in [35] — it may point to the importance of analiticity constraints in the calculation of the Compton amplitude. The data [43] for the photon asymmetry at $\theta_{\gamma}=90^{\circ}$ and energy $E_{\gamma}^{\text {lab }} \geq 500 \mathrm{MeV}$ are not reproduced by the calculation with the set "A". The description improves with the set "B" (dashed lines) though both parameter sets give very close results for cross sections (top panels). For the photon angle $120^{\circ}$ the difference between solid and dashed lines for $\Sigma_{\gamma}$ diminishes and the data do not allow to distinguish between the two parameter sets. The recoil-proton polarization $P_{y}^{p r}$ (Fig. (2), bottom) vanishes below the pion-production threshold since the imaginary part of the amplitude due to $\pi N$ rescattering is zero th. The behavior of $P_{y}^{p r}$ is in agreement with the present calculation as well as with the model [31], although the error bars on the data are large.

Above $\approx 900 \mathrm{MeV}$ the present model loses predictive power as tails of the higher resonances (not included in the model) will start to influence the cross section.

\section{RESULTS OF CALCULATIONS AND DISCUSSION}

\section{A. Cross sections for virtual Compton scattering}

Calculations of the exclusive differential cross sections for $\gamma p \rightarrow e^{-} e^{+} p$ at two photon energies are shown in Fig. (3). The energy $E_{\gamma}^{l a b}=320 \mathrm{MeV}$ corresponds to the $\Delta$ resonance, and $E_{\gamma}^{l a b}=700 \mathrm{MeV}$ roughly corresponds to the region of the $D_{13}(1520)$. At fixed energy the cross section depends on four essential variables. In presenting the results we will therefore fix some of them. The virtual-photon scattering angle $\theta_{\gamma}$ is chosen in the backward hemisphere as our calculations indicate that this provides favorable conditions to suppress the $\mathrm{BH}$ contribution.

In Fig. (3) the azimuthal lepton angle was fixed in plane, i.e. $\phi_{l}=0$ (coplanar kinematics). For the case when $e^{-}$and $e^{+}$are not distinguished the angle combination $\left\{\phi_{l}=0, \theta_{l}\right\}$ is equivalent to $\left\{\phi_{l}=\pi, \pi-\theta_{l}\right\}$. The results are shown as function of the polar angle $\theta_{l}$ at several values of the photon invariant mass.

The feature of the BH cross section (dashed lines) in coplanar kinematics is a pronounced peak which develops when either electron or positron moves along the direction of the initial photon. The propagator of the lepton in the $\mathrm{BH}$ amplitude in Fig. (1) (diagrams g, h) becomes large in this case. As is seen from Fig. (3), with increasing $M_{\gamma}$ the $\mathrm{BH}$ peak is shifted towards larger angles $\theta_{l}$, and for $M_{\gamma}=500 \mathrm{MeV}$ (Fig. (3), right, bottom) it occurs at

\footnotetext{
${ }^{4}$ Contribution to the imaginary part from the $\gamma N$ rescattering is of the order $e^{4}$ and thus negligibly small
} 
the angle $\theta_{B H}=32^{\circ}$. The maximal photon invariant mass at a given energy is determined from the relation $M_{\gamma}^{\max }=\sqrt{s}-m$ and corresponds to production of leptons with energies $\epsilon_{-}=\epsilon_{+}=\frac{1}{2} M_{\gamma}^{\max }$ and momenta $\vec{k}_{-}=-\vec{k}_{+}$. At this so-called kinematical limit the position of the peak reaches the maximal angle $\theta_{B H}^{\max }=\pi-\theta_{\gamma}$ which, for instance, for $E_{\gamma}^{\text {lab }}=700$ $\mathrm{MeV}$ and $\theta_{\gamma}=135^{\circ}$ takes the value $45^{\circ}$ at $M_{\gamma}^{\max }=543 \mathrm{MeV}$.

An example of a calculation in non-coplanar kinematics is shown in Fig. (仼). It is seen that the $\mathrm{BH}$ peak disappears as the momentum of $e^{-}$or $e^{+}$cannot be collinear to the photon momentum in this case. The $\phi_{l}$ dependence of the nuclear CS cross section is governed by the interference RFs which stand in front of $\cos \phi_{l}$ and $\cos 2 \phi_{l}$ in Eq. (11). The $\phi_{l}$ dependence of the $\mathrm{BH}$ contribution is more complicated.

At small invariant masses the nuclear CS cross section (Fig. (3), solid lines) shows a distinct peaking at forward and backward angles, while at large invariant masses it tends to flatten. This behavior can be understood from Eq. (10). Assuming that $\left(M_{\gamma} / 2 m_{e}\right)^{2} \gg 1$ one can show that the relative $e^{-} e^{+}$momentum obeys the condition

$$
|\vec{l}|=\left\{\begin{array}{cc}
\frac{1}{2} M_{\gamma}\left(\sin ^{2} \theta_{l}+M_{\gamma}^{2} / \vec{k}^{2}\right)^{-1 / 2}, & \text { if } M_{\gamma} \ll|\vec{k}|, \\
\frac{1}{2} M_{\gamma}, & \text { if } \quad|\vec{k}| \ll M_{\gamma} \sim M_{\gamma}^{\max } .
\end{array}\right.
$$

Therefore at small $M_{\gamma}$ the phase-space factor $|\vec{l}|^{3}$ rapidly increases as $\theta_{l}$ approaches $0^{\circ}$ or $180^{\circ}$. The functions $W_{T}$ and $W_{T T}$ become the dominant contributions in Eq. (11) and $\theta_{l}$ dependence of $\mathcal{S}$ is determined by $x_{T}$ and $x_{T T}$ :

$$
x_{T}=\frac{\sin ^{2} \theta_{l}+2 M_{\gamma}^{2} / \vec{k}^{2}}{2\left(\sin ^{2} \theta_{l}+M_{\gamma}^{2} / \vec{k}^{2}\right)}, \quad x_{T T}=\frac{\sin ^{2} \theta_{l}}{2\left(\sin ^{2} \theta_{l}+M_{\gamma}^{2} / \vec{k}^{2}\right)} .
$$

These are also rapidly changing functions, namely, $x_{T}$ increases from $1 / 2$ to 1 and $x_{T T}$ decreases from $1 / 2$ to 0 when $\theta_{l}$ approaches $0^{\circ}$ or $180^{\circ}$. The product $|\vec{l}|^{3} \mathcal{S}$ determines the behavior of the the cross section shown in the top panels of Fig. (3). At large invariant masses the phase-space factor is independent of the angle. The $\theta_{l}$ dependence of the cross section then comes only from the factors $x_{i}$ in Eqs. (12) and turns out to be rather flat for this particular kinematics.

Even if one moves away from the $\mathrm{BH}$ peak there is still a $\mathrm{BH}$ background. With increasing $M_{\gamma}$ its relative importance increases as is seen from Figs. (3, (4). Therefore one has to choose a relatively large $\theta_{\gamma}$ to obtain the situation in which the $\mathrm{BH}$ contribution can be ignored with respect to virtual CS. There is a relation between $M_{\gamma}$ and the angle $\theta_{\gamma}^{\min }$ which we define as the photon scattering angle, where virtual CS is larger than BH by approximately one order of magnitude. The dependence is shown in Table $\llbracket$ for two energies (for (almost) coplanar kinematics $\theta_{l}$ is taken to be larger than $\theta_{B H}$ to suppress the BH peak). As follows from Table $[$ favorable conditions in the $\Delta$ region are at invariant masses up to $\approx 200 \mathrm{MeV}$. At larger values of $M_{\gamma}$ the $\mathrm{BH}$ background becomes comparable to or larger than virtual CS at all angles $\theta_{\gamma}$. At an energy of $700 \mathrm{MeV}$ larger invariant masses up to $\approx 500 \mathrm{MeV}$ become accessible. 


\section{B. Results for response functions in virtual Compton scattering}

In Figs. (5-7) the response $W_{S}=W_{T}+W_{L}$ and the ratios of RFs (or elements of the density matrix defined in Eqs. (18.19)) are shown. The calculations are presented with the parameter set "B", as it gives better description of the photon asymmetry in real CS.

First one notices from the angular distributions that at $\theta_{\gamma}=0^{\circ}$ and $180^{\circ}$ the interference terms $\rho_{1-1}$ and $\rho_{10}$ vanish, that can be explained from rotation symmetry around the beam axis. This property serves as an additional test of the model.

In the $\Delta$ region (Fig. (5)) the transverse $\mathrm{RF}$ is dominant over the whole interval of $M_{\gamma}$. We studied the contribution of the additional coupling $G_{3}\left(k^{2}\right)$ in the $\gamma N \Delta$ vertex in Eq. (33) by varying $g_{3}$ within the limits $\pm g_{2}$. The effect turns out to be extremely small. This is due to small $k^{2} \leq 0.08 \mathrm{GeV}^{2}$ which can be reached at this energy, and the dominance of the

magnetic $f_{M M}^{1+}$ transition, which is very weakly dependent on $G_{3}\left(k^{2}\right)$. There is an effect of $\sigma$ exchange on the interference RFs, or on $\rho_{1-1}$ and $\rho_{10}$ (compare solid and dashed curves in Fig. (5)). This is interesting in view of the very small contribution of sigma exchange to the cross section near the $\Delta$ peak. However, the region of $M_{\gamma}$ above $\approx 150 \mathrm{MeV}$ will be difficult to access due to large $\mathrm{BH}$ contribution (see Table $\mathbb{\mathbb { Q }}$ ). The angular distribution of $W_{S}$ at fixed $M_{\gamma}$ is similar to real CS cross section $d \sigma(\gamma) / d \Omega_{\gamma}$, and is determined by the dominant $M 1$ multipole and its interference with $E 1$ and $E 2$ multipoles.

At a higher photon energy, shown in Figs. (6.7), the transverse response is still rather substantial. In Fig. (6) the effects of the important contributions are shown by switching off the corresponding process. The effect of the $D_{13}(1520)$ resonance is clearly seen in a pronounced angular distribution while other contributions give rise to a flat background (dotted lines) with $\Im \rho_{10}$ as an exception. This background in $\rho_{1-1}$ and $\Re \rho_{10}$ is also independent of $M_{\gamma}$ as is seen in the left panel. Other important contributions in this energy region are the $S_{11}(1535)$ excitation and the $\sigma$ exchange. The $S_{11}$ resonance contributes $10-20 \%$ to the cross section at $M_{\gamma}=300 \mathrm{MeV}$, and also to $\rho_{00}$ and $\Re \rho_{10}$ at backward angles; its role increases with increasing invariant mass. $\sigma$ exchange, as a t-channel contribution, shows up at backward angles with the exception of $\Im \rho_{10}$, where it is seen at forward angles. There is also a small contribution to $\rho_{1-1}$ from the $D_{33}(1700)$, however, its effect is not shown separately because the e.m. couplings of this resonance could not be fixed quite accurately from pion photoproduction. We should emphasize that all contributions add coherently in the total amplitude resulting in a strong interference. In particular, the $P_{33}(1232)$ resonance, through an interference, affects observables in the 2nd resonance region.

For comparison we also present calculations with the parameter set "A" (long-dashed lines). In $W_{S}$ large differences between the set "A" and set "B" appear at $\theta_{\gamma}<90^{\circ}$ and large invariant masses, on average the differences are about $20 \%$. The most striking effects can be observed in $\rho_{00}, \rho_{1-1}$ and $\Re \rho_{10}$ (compare solid and long-dashed lines in Fig. (6)). The big difference in $\rho_{1-1}$ at $90^{\circ}$ is similar to that for the photon asymmetry in Fig. (2), while at larger angles the difference is reduced. As is seen from Fig. (6) for $\rho_{00}$ and $\Re \rho_{10}$, the longitudinal response also proves to be rather sensitive to parameters $a_{1,2}$ for the $D_{13}$.

In principle one might expect a contribution of the Roper resonance, $P_{11}(1440)$, to the longitudinal response. Data on pion electroproduction (see review [45]) indicate however that the corresponding helicity amplitude $S_{1 / 2}$ is very small, consistent with zero. The longitudinal coupling in the $\gamma N P_{11}$ vertex has thus been neglected. The transverse coupling 
is known to be small from real-photon data.

In Fig. (7) the effect of $G_{3}\left(k^{2}\right)$ in the $\gamma N D_{13}(1520)$ vertex is demonstrated. The dashed curves are calculated with $g_{3}=-3.3$ which is chosen to reproduce the $k^{2}$ dependence of the ratio of the helicity amplitudes $A_{1 / 2}$ and $A_{3 / 2}$ (or the corresponding multipoles $E_{2-}^{1 / 2}$ and $M_{2-}^{1 / 2}$ ) for pion electroproduction [45]. As is seen, the $G_{3}\left(k^{2}\right)$ coupling has a considerable effect on $W_{S}$ and thus on the cross section. The reason is that $G_{3}\left(k^{2}\right)$ strongly influences the electric dipole transition $E 1$ and the latter in turn becomes the dominant term at large positive $k^{2}$. For the following consideration we choose $\sqrt{s}=M_{R}$ ( $M_{R}$ is a resonance mass), then the 3-momentum squared of the virtual photon is

$$
\vec{k}^{2}=k_{0}^{2}-k^{2}=\frac{1}{4 M_{R}^{2}}\left[\left(M_{R}+m\right)^{2}-k^{2}\right]\left[\left(M_{R}-m\right)^{2}-k^{2}\right] .
$$

It is seen that $\vec{k}^{2}$ decreases in the time-like region and vanishes at the kinematical limit, where $k_{0}=M_{\gamma}^{\max }=M_{R}-m$.

The behavior of RFs for $e^{-} e^{+}$production can be understood based on general properties of the resonance multipoles at small $|\vec{k}| \ll R^{-1}$, where $R$ is a typical interaction radius. According to [46] (ch. 6.2 and 6.3) one has for the e.m. transition with multipolarity $j$

$$
T(\vec{k} ; M j) \propto|\vec{k}|^{j}, \quad T(\vec{k} ; E j) \propto|\vec{k}|^{j-1} k_{0}, \quad T(\vec{k} ; L j) \simeq-T(\vec{k} ; E j)\left(\frac{j}{j+1}\right)^{1 / 2} .
$$

Therefore for the $D_{13}$ resonance magnetic quadrupole $(M 2)$ vanishes, and electric dipole $(E 1)$ and longitudinal dipole $(L 1)$ remain finite at $k^{2}=\left(M_{\gamma}^{\max }\right)^{2}$. At the same time all multipoles for the $P_{33}(1232)$ resonance, magnetic dipole $(M 1)$, electric and longitudinal quadrupoles ( $E 2$ and $L 2$ ), are proportional to $|\vec{k}|$ and go to zero. To have a more quantitative estimate we can use predictions of the non-relativistic quark model (e.g., Ref. [47]). In Table [1] expressions are collected for the e.m. helicity amplitudes and the resonance pion-production multipoles. It follows from Table [1 that:

i) $A_{1 / 2}$ and $A_{3 / 2}$ for the $D_{13}$ are finite at small $|\vec{k}|$, while those for the $P_{33}$ vanish;

ii) if $|\vec{k}| \rightarrow 0$ the ratio $A_{1 / 2} / A_{3 / 2}$ for the $D_{13}$ takes the value $1 / \sqrt{3}$ which coincides with the corresponding ratio for the $P_{33}$ (in this and other $S U(6)$ symmetrical models);

iii) as $k^{2}$ increases towards the maximal value the spin-flip contribution (terms $\propto \vec{k}^{2}$ ) diminishes for the $D_{13}$, correspondingly $E_{2-}$ increases and $M_{2-}$ decreases (absolute values).

Since the $E 1$ transition dominates the $D_{13} \rightarrow \gamma^{*} N$ process, the coupling $G_{3}\left(k^{2}\right)$ affects strongly the cross section in Fig. (7). The transverse response $W_{T}$ at the resonance position is roughly proportional to $|E 1|^{4}$, while the longitudinal response $W_{L}$ to $|L 1|^{2}|E 1|^{2}$. At small $|\vec{k}|$ one can make use of Siegert's theorem (last relation in Eqs. (37)) which relates $L 1$ and $E 1$ amplitudes. From this it can be seen that the ratio $\rho_{00}=W_{L} /\left(W_{T}+W_{L}\right)$ is equal to $1 / 3$, irrespective of $G_{3}\left(k^{2}\right)$. Other elements of the density matrix on Fig. (7), except $\Im \rho_{10}$, show a dependence on $G_{3}\left(k^{2}\right)$.

It is of interest to compare Figs. (6.7) for the $D_{13}$ with Fig. (50) for the $P_{33}$ resonance. Due to a decrease of the $M 1$ intensity as a function of $M_{\gamma}$ the response $W_{S}$ for the $\Delta$ resonance falls off towards the maximal $M_{\gamma}$. The longitudinal response $W_{L}$ contains the small resonance multipole $L 2$ which also vanishes at $|\vec{k}| \rightarrow 0$, while the background receives nonvanishing contributions from $L 1$ multipoles (such as $L_{0+}$ and $L_{2-}$ in pion electroproduction). These 
cause an increase of $\rho_{00}$ at large photon masses in Fig. (5). This effect comes as a result of a balance between $G_{1}\left(k^{2}\right)$ and $G_{2}\left(k^{2}\right)$ contributions in the $\gamma N \Delta$ vertex in Eq. (33). Note also that in the quark model in Table $\mathbb{T}$ the electric quadrupole is absent, $E 2=L 2=0$, which corresponds to a particular choice of the couplings $2 m g_{1}=M_{\Delta} g_{2}=M_{\Delta} g_{3}$.

The features of the e.m decay of the resonances in the time-like region are, in general, different from those studied in electron scattering, where $|\vec{k}|$ is always larger than $k_{0}$. For instance, in pion photo- and electroproduction interesting properties of the $D_{13}(1520)$ resonance have been observed at $-3 \mathrm{GeV}^{2}<k^{2} \leq 0$ (see, e.g. [45]). In terms of the multipoles, the ratio $E 1 / M 2 \approx-1$ at large negative $k^{2}$, crosses zero at $k^{2} \approx-1 \mathrm{GeV}^{2}$ and is about +2.1 at the real-photon point. It would be of interest to see if this ratio keeps on increasing at positive $k^{2}$ and becomes very large near $k^{2}=\left(M_{D_{13}}-m\right)^{2}=0.3364 \mathrm{GeV}^{2}$.

Finally we note that the increase of $W_{S}$ in Figs. (6.7) above $M_{\gamma} \approx 500 \mathrm{MeV}$ is due to the fact that the e.m. form factors contain the $\rho$-meson propagator. As $M_{\gamma}$ approaches the $\rho$-meson pole (which would be possible at photon energies above $1 \mathrm{GeV}$ ), the process $\gamma p \rightarrow e^{-} e^{+} p$ proceeds through creation of the $\rho$ meson, as assumed in VMD models.

\section{CONCLUSIONS}

We investigated virtual Compton scattering on the proton in the time-like region $(\gamma p \rightarrow$ $\left.e^{-} e^{+} p\right)$. When in an experiment the $e^{-}$and $e^{+}$are not distinguished, the Bethe-Heitlernuclear interference vanishes and the cross section is the incoherent sum of cross sections for the $\mathrm{BH}$ and the nuclear processes. We have shown that, contrary to common preconception, a considerable part of the phase space, mainly at backward angles for the virtual photon, is hardly contaminated by the Bethe-Heitler process. Under these conditions it is possible to decompose the exclusive cross section in terms of response functions. These are directly related to the photon density matrix $\rho_{\lambda \lambda^{\prime}}$ which characterizes polarization properties of the virtual photon. This offers a possibility to analyze $e^{-} e^{+}$production experiments also in terms of matrix elements $\rho_{\lambda \lambda^{\prime}}$ or, equivalently, tensor and vector polarizations of the timelike photon.

Differential cross sections and matrix elements $\rho_{\lambda \lambda^{\prime}}$ are calculated in a unitary K-matrix model which includes nucleon, mesons, and baryon resonances with masses up to $1.7 \mathrm{GeV}$. The model is tested for real-photon Compton scattering at energies up to $1 \mathrm{GeV}$. The agreement with data is reasonable and of a comparable quality to recent K-matrix calculation [35] and dispersion-relation analysis [31].

The dilepton production in the $\Delta$-resonance region is dominated by the transverse response. At the largest $M_{\gamma}$ at this energy certain elements $\rho_{\lambda \lambda^{\prime}}$ show the effect of $\sigma$ exchange. The latter could be an approximation to various t-channel scalar-isoscalar exchanges (e.g., two-pion, $\epsilon(760)$ and $\left.f_{0}(400-900)\right)$.

Photon energies corresponding to the 2 nd resonance region, of about $700 \mathrm{MeV}$, allow one to explore higher photon masses up to $\approx 500 \mathrm{MeV}$. Main mechanisms contributing here are the $D_{13}(1520)$-resonance excitation and, to a lesser extent, the $S_{11}(1535)$ resonance and $\sigma$ exchange. The transverse-transverse element $\rho_{1-1}$ strongly depends on the so-called off-shell parameters $a_{1}$ and $a_{2}$ in the e.m. vertex of the $D_{13}$. The longitudinal $\rho_{00}$ and longitudinaltransverse $\Re \rho_{10}$ elements turn out to be sensitive to all contributions and with increasing photon invariant mass this sensitivity becomes more pronounced. At large $M_{\gamma}$ the cross 
section and the density matrix become strongly dependent on the part of the e.m. vertex of the $D_{13}(1520)$ which contributes only for virtual photons.

Response functions are thus shown to be an important tool to distinguish between different mechanisms in the $e^{-} e^{+}$production in the resonance region. If the resonance contributions can be separated, this will allow for studying their e.m. properties in the time-like region which may give an information supplementary to that obtained from the electron scattering.

\section{ACKNOWLEDGMENTS}

This work is supported by the Foundation for Fundamental Research of the Netherlands (NWO). A. Yu. K. acknowledges a special grant from the NWO. The authors thank A. I. L'vov for sending results of calculations in the dispersion-relation approach. We thank also J. Bacelar for stimulating discussions on the experimental feasibility of virtual Compton scattering and valuable suggestions. Discussions with J. Messchendorp, M. Mostovoy and R. Timmermans are highly appreciated.

\section{APPENDIX A: RESPONSE FUNCTIONS IN HELICITY FORMALISM}

It is convenient to introduce the following set of polarization vectors for a time-like photon

$$
\epsilon^{*}(0)=\frac{1}{M_{\gamma}}\left(|\vec{k}|, 0,0, k_{0}\right), \quad \epsilon^{*}( \pm 1)=\frac{1}{\sqrt{2}}(0, \mp 1, i, 0)
$$

which satisfy $\epsilon^{*}(\lambda) \cdot k=0$ and

$$
\epsilon^{*}(\lambda) \cdot \epsilon\left(\lambda^{\prime}\right)=-\delta_{\lambda \lambda^{\prime}}, \quad \sum_{\lambda=0, \pm 1} \epsilon^{* \mu}(\lambda) \epsilon^{\nu}(\lambda)=-g^{\mu \nu}+\frac{k^{\mu} k^{\nu}}{M_{\gamma}^{2}} .
$$

The RFs defined in Eq. (13) can now be written as

$$
\begin{aligned}
W_{T} & =\frac{1}{4} \sum_{\text {polar. }} \quad\left|J \cdot \epsilon^{*}(+1)\right|^{2}+\left|J \cdot \epsilon^{*}(-1)\right|^{2}=\frac{1}{4} \sum_{\Lambda, \Lambda^{\prime}= \pm 1 / 2, \lambda= \pm 1}\left|f_{+1 \Lambda^{\prime}, \lambda \Lambda}\right|^{2}+\left|f_{-1 \Lambda^{\prime}, \lambda \Lambda}\right|^{2}, \\
W_{L} & =\frac{1}{4} \sum_{\text {polar. }} \quad\left|J \cdot \epsilon^{*}(0)\right|^{2}=\frac{1}{4} \sum_{\Lambda, \Lambda^{\prime}= \pm 1 / 2, \lambda= \pm 1}\left|f_{0 \Lambda^{\prime}, \lambda \Lambda}\right|^{2}, \\
W_{T T} & =\frac{1}{4} \sum_{\text {polar. }} \quad 2 \Re J \cdot \epsilon^{*}(+1)\left[J \cdot \epsilon^{*}(-1)\right]^{*}=\frac{1}{4} \sum_{\Lambda, \Lambda^{\prime}= \pm 1 / 2, \lambda= \pm 1} 2 \Re f_{+1 \Lambda^{\prime}, \lambda \Lambda} f_{-1 \Lambda^{\prime}, \lambda \Lambda}^{*}, \\
W_{L T}=\frac{1}{4} \sum_{\text {polar. }} \quad 2 \Re J \cdot \epsilon^{*}(0)\left[J \cdot \epsilon^{*}(+1)-J \cdot \epsilon^{*}(-1)\right]^{*} & \\
= & \frac{1}{4} \sum_{\Lambda, \Lambda^{\prime}= \pm 1 / 2, \lambda= \pm 1} 2 \Re f_{0 \Lambda^{\prime}, \lambda \Lambda}^{*}\left[f_{+1 \Lambda^{\prime}, \lambda \Lambda}-f_{-1 \Lambda^{\prime}, \lambda \Lambda}\right],
\end{aligned}
$$

where we introduced the helicity amplitude 


$$
f_{\lambda^{\prime} \Lambda^{\prime}, \lambda \Lambda}=e \epsilon_{\mu}^{*}\left(\lambda^{\prime}\right) \bar{u}\left(p^{\prime}, \Lambda^{\prime}\right) M^{\mu \nu} u(p, \Lambda) \epsilon_{\nu}(\lambda)
$$

which is a function of $s, \theta_{\gamma}$ and $M_{\gamma}$. The additional RF $\tilde{W}_{L T}$, which defines the vector polarization $p_{y}=i \frac{2}{\sqrt{3}} t_{11}$ of the virtual photon (see Eq. (24)), can be calculated using the last formula in Eq. (A3), where the operation $\Re \ldots$ should be replaced by $\Im . .$.

Due to space-reflection invariance there are 8 (4) independent transverse (longitudinal) amplitudes in the sums in Eqs. (A3). They can be chosen according to Table [1]. The remaining amplitudes are determined from the relation $f_{-\lambda^{\prime}-\Lambda^{\prime},-\lambda-\Lambda}=(-1)^{\lambda^{\prime}-\Lambda^{\prime}-(\lambda-\Lambda)} f_{\lambda^{\prime} \Lambda^{\prime}, \lambda \Lambda}$ 48.

In terms of $f_{i}$ of Table III one can rewrite the response functions as

$$
\begin{aligned}
W_{T} & =\frac{1}{2} \sum_{i=1}^{8}\left|f_{i}\right|^{2}, \quad W_{L}=\frac{1}{2} \sum_{i=9}^{12}\left|f_{i}\right|^{2}, \\
W_{T T} & =\Re\left(f_{1} f_{3}^{*}+f_{2} f_{4}^{*}+f_{5} f_{7}^{*}+f_{6} f_{8}^{*}\right), \\
W_{L T} & =\Re\left[f_{9}^{*}\left(f_{1}-f_{3}\right)-f_{10}^{*}\left(f_{2}-f_{4}\right)+f_{11}^{*}\left(f_{5}-f_{7}\right)-f_{12}^{*}\left(f_{6}-f_{8}\right)\right] .
\end{aligned}
$$

For real photons due to the time reversal [48] one has in addition $f_{7}^{r}=f_{3}^{r}$ and $f_{8}^{r}=-f_{4}^{r}$, where the superscript " $r$ " indicates the real CS limit: $f_{\lambda^{\prime} \Lambda^{\prime}, \lambda \Lambda}^{r}\left(s, \theta_{\gamma}\right)=f_{\lambda^{\prime} \Lambda^{\prime}, \lambda \Lambda}\left(s, \theta_{\gamma}, M_{\gamma}=0\right)$. In this case

$$
\begin{aligned}
& W_{T}^{r}=\frac{1}{2}\left[\left|f_{1}^{r}\right|^{2}+\left|f_{2}^{r}\right|^{2}+2\left|f_{3}^{r}\right|^{2}+2\left|f_{4}^{r}\right|^{2}+\left|f_{5}^{r}\right|^{2}+\left|f_{6}^{r}\right|^{2}\right], \\
& W_{T T}^{r}=\Re\left[f_{3}^{r *}\left(f_{1}^{r}+f_{5}^{r}\right)+f_{4}^{r *}\left(f_{2}^{r}-f_{6}^{r}\right)\right]
\end{aligned}
$$

since $f_{9}^{r}, \cdots, f_{12}^{r}$ vanish. Eq. (A6) agrees with definitions given in [24, [0] up to a common normalization factor.

In addition to the photon asymmetry in Eq. (29) we will also calculate the polarization of the recoil proton in real CS. It can be expressed through the helicity amplitudes as

$$
\begin{array}{rl}
P_{y}^{p r}=\frac{1}{4 W_{T}^{r}} \sum_{\Lambda= \pm 1 / 2, \lambda, \lambda^{\prime}= \pm 1} & 2 \Im f_{\lambda^{\prime}-1 / 2, \lambda \Lambda}^{r} f_{\lambda^{\prime}+1 / 2, \lambda \Lambda}^{r *} \\
= & -\frac{1}{W_{T}^{r}} \Im\left[f_{4}^{r *}\left(f_{1}^{r}+f_{5}^{r}\right)+f_{3}^{r *}\left(f_{6}^{r}-f_{2}^{r}\right)\right] .
\end{array}
$$




\section{REFERENCES}

[1] Proceedings of Workshop on Virtual Compton Scattering, Clermont-Ferrand, France, 1996.

[2] P. A. M. Guichon and M. Vanderhaeghen, Progr. Part. Nucl. Phys. 41, 125 (1998).

[3] M. Schäfer, H. C. Dönges, and U. Mosel, Phys. Lett. B 342, 13 (1995).

[4] H. Alvensleben, U. Becker, P. J. Biggs et al., Phys. Rev. Lett. 30, 328 (1973).

[5] A. I. L'vov, S. Scopetta, D. Drechsel, and S. Scherer, in Ref. [四] p.186; Phys. Rev. C 57, 312 (1998).

[6] A. Yu. Korchin, O. Scholten, and F. de Jong, Phys. Lett. B 402, 1 (1997).

[7] A. E. L. Dieperink and S. I. Nagorny, Phys. Lett. B 397, 20 (1997).

[8] J. C. S. Bacelar, private communication.

[9] J. D. Bjorken, S. D. Drell, and S. C. Frautschi, Phys. Rev. 112, 1409 (1958).

[10] T. W. Donnely, in New Vistas in Electro-Nuclear Physics, ed. by E. L. Tomusiak, H. S. Caplan, and E. T. Dressler (Plenum Press, New York, 1986), p.151.

[11] K. Schilling, R. Seyboth, and G. Wolf, Nucl. Phys. B15, 397 (1970).

[12] O. Scholten, A. Yu. Korchin, V. Pascalutsa, and D. Van Neck, Phys. Lett. B 384, 13 (1996).

[13] A. Yu. Korchin, O. Scholten, and R. G. E. Timmermans, Phys. Lett. B 438, 1 (1998).

[14] C. Itzykson and J. -B. Zuber, Quantum Field Theory (McGraw-Hill Book Company, 1980).

[15] D. Van Neck, A. E. L. Dieperink, and O. Scholten, Nucl. Phys. A574, 643 (1994).

[16] A. Yu. Korchin, D. Van Neck, M. Waroquier, O. Scholten, and A. E. L. Dieperink, Phys. Lett. B 441, 17 (1998).

[17] A. Yu. Korchin and O. Scholten, Nucl. Phys. A581, 493 (1995).

[18] J. G. Messchendorp, J. C. S. Bacelar, M. J. van Goethem et al., Phys. Rev. Lett. 83, 2530 (1999); Phys. Rev. C, in press.

[19] J. Daboul, Nucl. Phys. B4, 180 (1967).

[20] W. M. Kloet, Wen-Tai Chiang, and Frank Tabakin, Phys. Rev. C 58, 1086 (1998).

[21] W. M. Kloet and Frank Tabakin, Phys. Rev. C 61, 015501 (2000).

[22] H. M. Pilkuhn, Relativistic Particle Physics (Springer-Verlag, New-York - Heidelberg Berlin, 1979).

[23] B. A. Robson, The Theory of Polarization Phenomena (Clarendon Press, Oxford, 1974).

[24] W. Pfeil, H. Rollnik, and S. Stankowski, Nucl. Phys. B73, 166 (1974).

[25] D. Drechsel and L. Tiator, J. Phys. G: Nucl. Phys. 18, 449 (1992).

[26] R. D. Peccei, Phys. Rev. 181, 1902 (1969).

[27] M. G. Olsson and E. T. Osypowski, Nucl. Phys. B87, 399 (1975).

[28] M. Benmerrouche, R. M. Davidson, and N. C. Mukhopadhyay, Phys. Rev. C 39, 2339 (1989).

[29] V. Pascalutsa and O. Scholten, Nucl. Phys. A591, 658 (1995).

[30] G. E. Brown, Mannque Rho, and W. Weise, Nucl. Phys. A454, 669 (1986); M. Gari and W. Krumpelmann, Z. Phys. A 322, 689 (1985); R. Williams, S. Krewald, and K. Linen, Phys. Rev. C 51, 566 (1995).

[31] A. I. L'vov, V. A. Petrun'kin, and M. Schumacher, Phys. Rev. C 55, 359 (1997); A. I. L'vov and A. M. Nathan, Phys. Rev. C 59, 1064 (1999), and private communication. In 
the calculation shown in Fig. (2) the solution SM99K of the VPI analysis [32] has been used.

[32] Virginia Tech SAID Facility, see http://said.phys.vt.edu/analysis; R. A. Arndt, I. I. Strakovskii, and R. L. Workman, Phys. Rev. C 53, 430 (1996).

[33] A. Hünger, J. Peise, A. Robbiano et al., Nucl. Phys. A620, 385 (1997).

[34] D. Drechsel, M. Gorchtein, B. Pasquini, and M. Vanderhaeghen, Phys. Rev. C 61, 015204 (2000).

[35] T. Feuster and U. Mosel, Phys. Rev. C 59, 460 (1999).

[36] J. W. DeWire, M. Feldman, V. L. Highland, and R. Littauer, Phys. Rev. 124, 909 (1961).

[37] R. F. Stiening, E. Loh, and M. Deutsch, Phys. Rev. Lett. 10, 536 (1963).

[38] H. Genzel, M. Jung, R. Wedemeyer, and H. J. Weyer, Z. Phys. A 279, 399 (1976).

[39] K. Toshioka, M. Chiba, S. Kato et al., Nucl. Phys. B141, 364 (1978).

[40] T. Ishii, K. Egawa, S. Kato et al., Nucl. Phys. B165, 189 (1980).

[41] P. S. Baranov, V. A. Kuznetsova, L. I. Slovokhotov, G. A. Sokol, and L. N. Shtarkov, Sov. J. Nucl. Phys. 3, 791 (1966).

[42] G. Blanpied, M. Blecher, A. Caracappa et al., Phys. Rev. Lett. 76, 1023 (1996).

[43] F. V. Adamian, A. Yu. Buniatian, G. S. Frangulian et al., J. Phys. G: Nucl. Part. Phys. 19, L139 (1993).

[44] Y. Wada, K. Egawa, A. Imanishi, T. Ishii, S. Kato, K. Ukai, F. Naito, H. Hara, T. Noguchi, and K. Takahashi, Nucl. Phys. B247, 313 (1984).

[45] V. D. Burkert, Czech. J. Phys. 46, 627 (1996).

[46] J. M. Eisenberg and W. Greiner, Excitation mechanisms of the nucleus (North-Holland Publishing Company, Amsterdam-London, 1970).

[47] R. Bijker, F. Iachello, and A. Leviatan, Phys. Rev. C 54, 1935 (1996).

[48] M. Jacob and G. C. Wick, Ann. Phys. (N.Y.) 7, 404 (1959). 


\section{TABLES}

\begin{tabular}{|c|c|c|c|}
\hline \multicolumn{2}{|c|}{$E_{\gamma}^{l a b}=320 \mathrm{MeV}\left(M_{\gamma}^{\max }=278 \mathrm{MeV}\right)$} & $E_{\gamma}^{l a b}=700 \mathrm{MeV}\left(M_{\gamma}^{\max }=543 \mathrm{MeV}\right)$ & $\begin{array}{l}x=543 \mathrm{MeV}) \\
\theta_{\gamma}^{\min }(\mathrm{deg})\end{array}$ \\
\hline 5 & 30 & 5 & 30 \\
\hline 50 & 90 & 50 & 60 \\
\hline 100 & 120 & 100 & 70 \\
\hline 150 & 150 & 150 & 90 \\
\hline \multirow[t]{4}{*}{200} & 170 & 250 & 110 \\
\hline & & 350 & 130 \\
\hline & & 450 & 150 \\
\hline & & 530 & 170 \\
\hline
\end{tabular}

TABLE I. Minimal photon angle (defined in sect. [IIA) as function of photon invariant mass.

\begin{tabular}{c|cc}
\hline \hline & $D_{13}(1520), \mathrm{I}=1 / 2$ (proton) & $P_{33}(1232), \mathrm{I}=3 / 2$ \\
\hline$\left(\frac{k_{0}}{\pi}\right)^{1 / 2} A_{1 / 2}$ & $2 i\left(\vec{k}^{2}-b^{2}\right) a F\left(\vec{k}^{2}\right) \mu_{q}$ & $-\frac{2 \sqrt{2}}{3}|\vec{k}| F\left(\vec{k}^{2}\right) \mu_{q}$ \\
$\left(\frac{k_{0}}{\pi}\right)^{1 / 2} A_{3 / 2}$ & $-2 i \sqrt{3} b^{2} a F\left(\vec{k}^{2}\right) \mu_{q}$ & $-\frac{2 \sqrt{2}}{\sqrt{3}}|\vec{k}| F\left(\vec{k}^{2}\right) \mu_{q}$ \\
$A_{1 / 2} / A_{3 / 2}$ & $\frac{1}{\sqrt{3}}\left(1-\frac{\vec{k}^{2}}{b^{2}}\right)$ & $\frac{1}{\sqrt{3}}$ \\
$E_{2-}^{I}(E 1)$ & $\frac{i}{\sqrt{3}}\left(\vec{k}^{2}-4 b^{2}\right) a F\left(\vec{k}^{2}\right) \mu_{q} f$ & - \\
$E_{1+}^{I}(E 2)$ & - & 0 \\
$M_{1+}^{I}(M 1)$ & - & $\frac{4}{\sqrt{3}}|\vec{k}| F\left(\vec{k}^{2}\right) \mu_{q} f$ \\
$M_{2-}^{I}(M 2)$ & $-\frac{i}{\sqrt{3}} \vec{k}^{2} a F\left(\vec{k}^{2}\right) \mu_{q} f$ & - \\
\hline \hline
\end{tabular}

TABLE II. Electromagnetic helicity amplitudes and pion-production multipoles for $D_{13}(1520)$ and $P_{33}(1232)$ resonances in model [47]. $F\left(\vec{k}^{2}\right)=\left(1+a^{2} \vec{k}^{2}\right)^{-2}, b^{2}=m_{q} k_{0} / g_{q}$, and $m_{q}, \mu_{q}, g_{q}$ and $a$ are respectively constituent quark mass, scale magnetic moment, gyromagnetic ratio, and scale parameter. The constant $f=\left(\pi / k_{0}\right)^{1 / 2}\left(k_{\gamma} m \Gamma_{\pi N} / 4 \pi q_{\pi} M_{R} \Gamma_{\text {tot }}^{2}\right)^{1 / 2}$ relates helicity amplitudes to e.m. multipoles, where $k_{\gamma}$ is equivalent real-photon energy, $q_{\pi}$ is pion 3 -momentum, $\Gamma_{\pi N}\left(\Gamma_{t o t}\right)$ is one-pion (total) decay width of the resonance.

\begin{tabular}{ll|llll}
\hline \hline$f_{1}$ & $f_{+1+1 / 2,+1+1 / 2}$ & $f_{5}$ & $f_{+1-1 / 2,+1-1 / 2}$ & $f_{9}$ & $f_{0+1 / 2,+1+1 / 2}$ \\
$f_{2}$ & $f_{-1-1 / 2,+1+1 / 2}$ & $f_{6}$ & $f_{-1+1 / 2,+1-1 / 2}$ & $f_{10}$ & $f_{0-1 / 2,+1+1 / 2}$ \\
$f_{3}$ & $f_{-1+1 / 2,+1+1 / 2}$ & $f_{7}$ & $f_{-1-1 / 2,+1-1 / 2}$ & $f_{11}$ & $f_{0-1 / 2,+1-1 / 2}$ \\
$f_{4}$ & $f_{+1-1 / 2,+1+1 / 2}$ & $f_{8}$ & $f_{+1+1 / 2,+1-1 / 2}$ & $f_{12}$ & $f_{0+1 / 2,+1-1 / 2}$ \\
\hline \hline
\end{tabular}

TABLE III. Independent helicity amplitudes in the $\gamma p \rightarrow e^{-} e^{+} p$ reaction. 


\section{FIGURES}
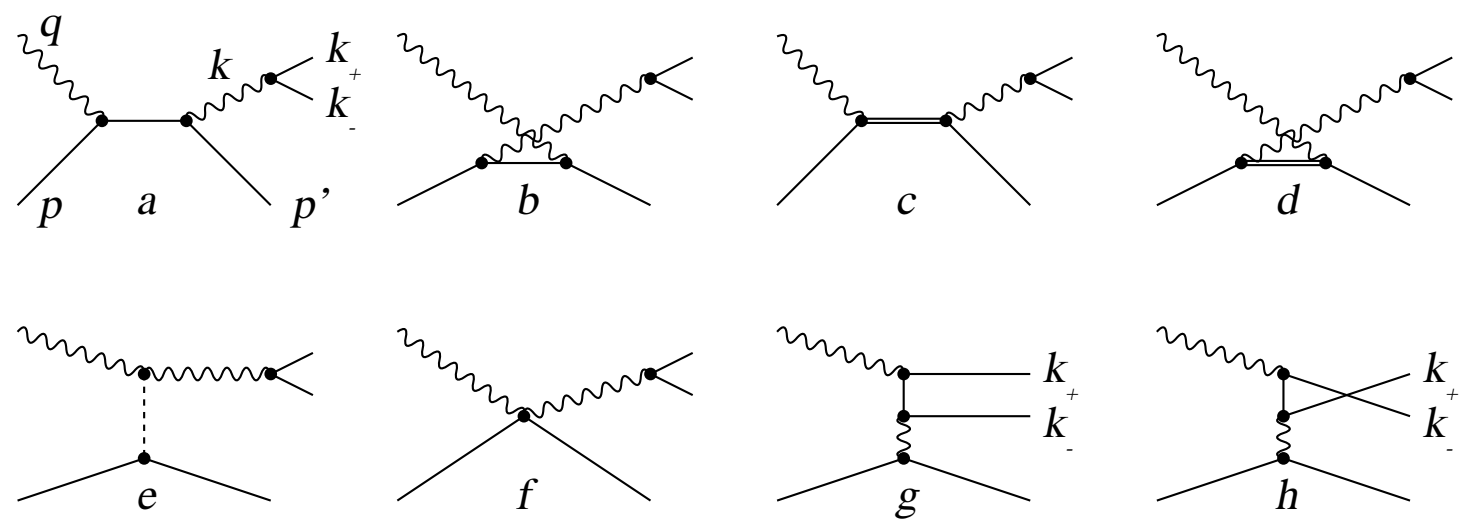

FIG. 1. Tree-level graphs describing the $\gamma N \rightarrow e^{-} e^{+} N$ process. Diagrams a $-\mathrm{f}$ correspond to virtual $\mathrm{CS}$ on the nucleon, g and $\mathrm{h}$ to the Bethe-Heitler contribution. Single (double) solid line depicts a nucleon (baryon resonance), wavy line a photon, dashed line $\pi^{0}, \sigma$ and $\eta$ mesons. Diagram $\mathrm{f}$ describes a possible contact $\gamma \gamma^{*} N N$ vertex. 


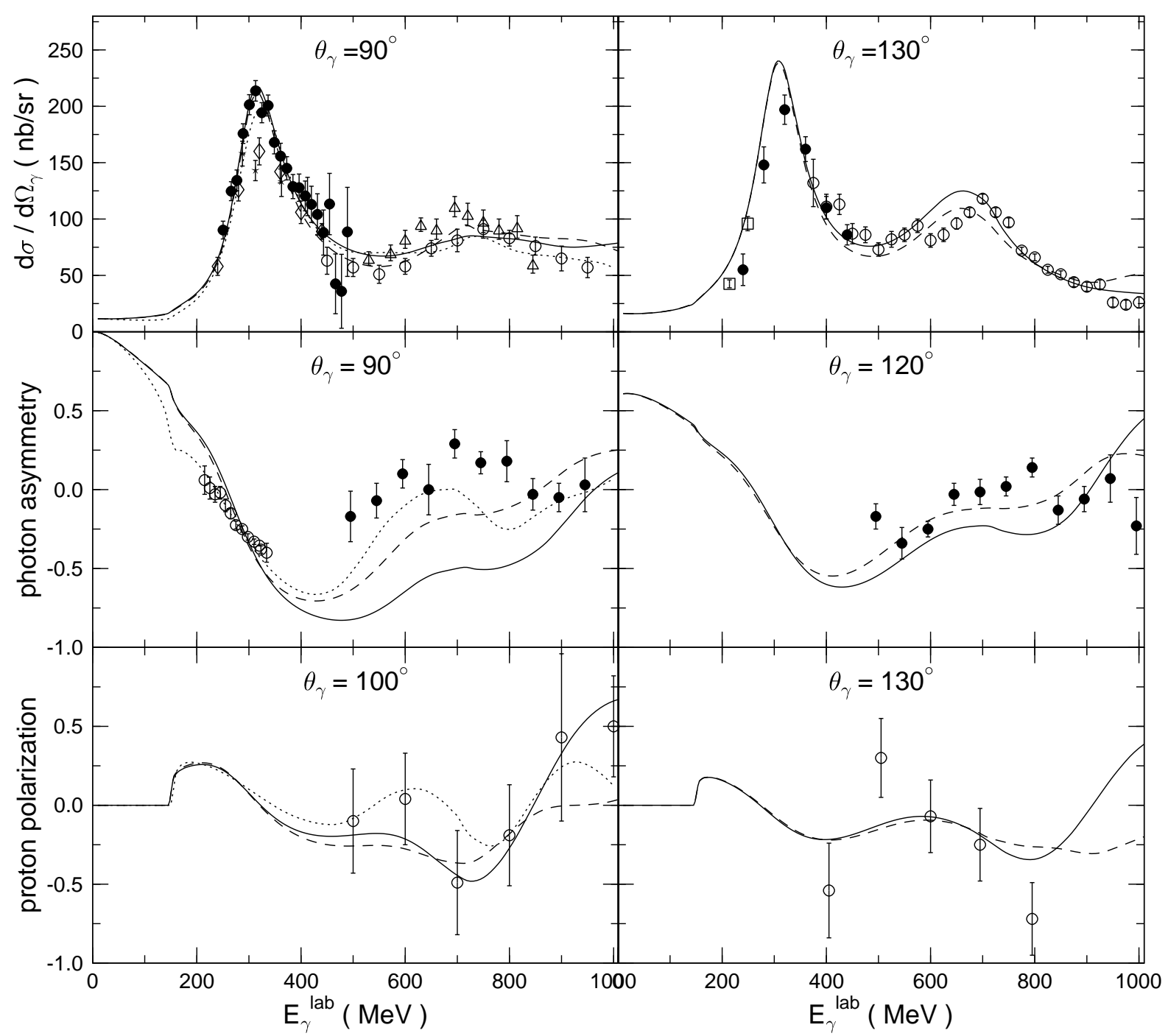

FIG. 2. Differential cross section (top), photon asymmetry (middle), and recoil-proton polarization (bottom) for real-photon Compton scattering as function of photon energy. Solid (dashed) lines are the present model calculations with the parameter set "A" ("B"), dotted lines are calcu-

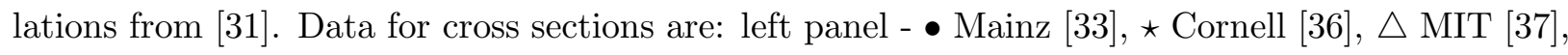
$\diamond$ Bonn [38], ○ Tokyo [39]; right panel - • Bonn [38], ○ Tokyo 40], $\square$ Moscow (Lebedev Inst.) [41]. Data for photon asymmetry: ○ LEGS Collaboration [42], • Erevan 443; and for proton polarization: $\circ$ Tokyo [44. 

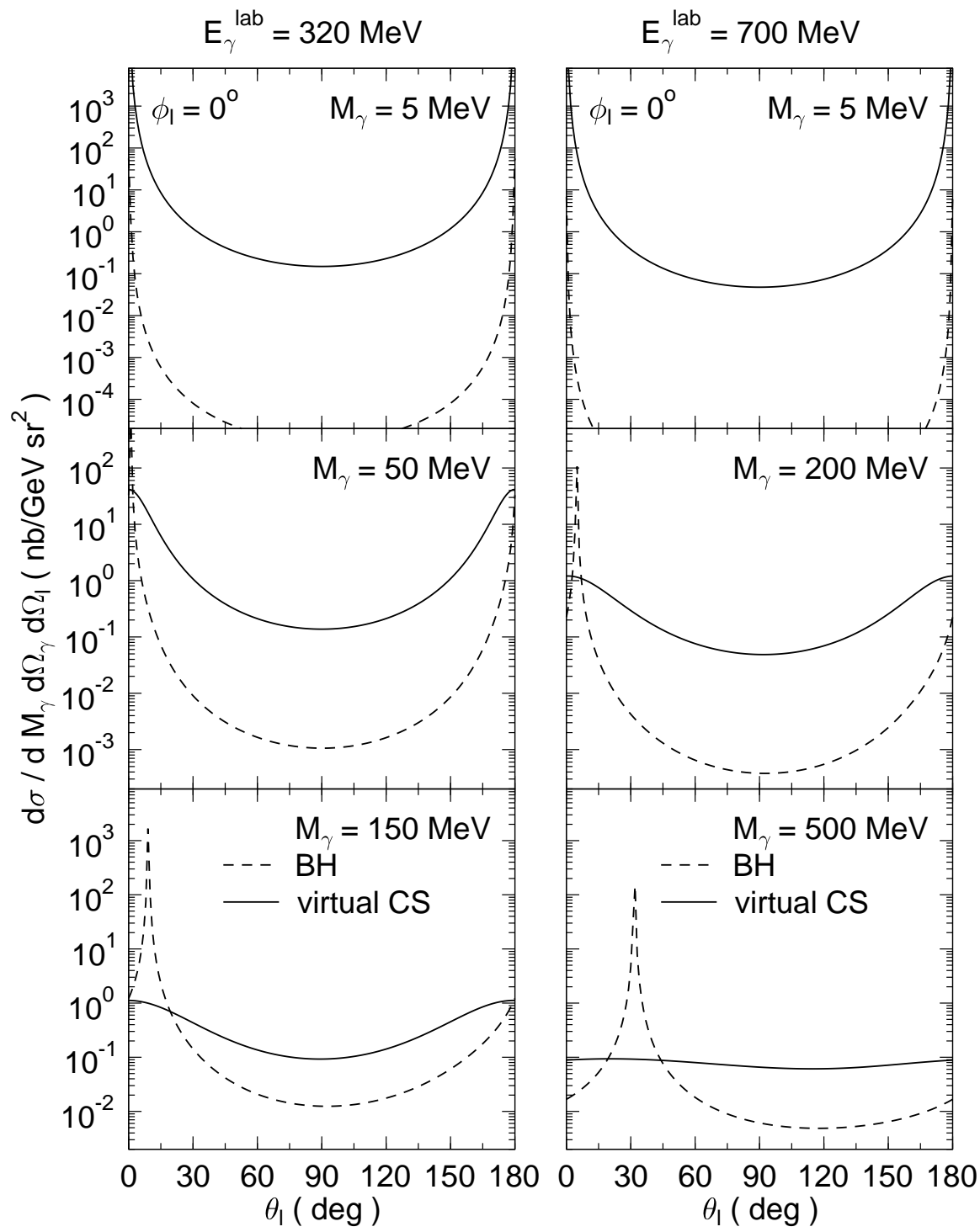

FIG. 3. Exclusive differential cross section in the c.m. as function of lepton "sharing" angle $\theta_{l}$ in coplanar kinematics. Left panel corresponds to $E_{\gamma}^{l a b}=320 \mathrm{MeV}$, right panel to $E_{\gamma}^{l a b}=700 \mathrm{MeV}$. The virtual-photon angle is $\theta_{\gamma}=135^{\circ}$. Dashed and solid lines are respectively the Bethe-Heitler and the virtual Compton scattering (parameter set "A") contributions. 


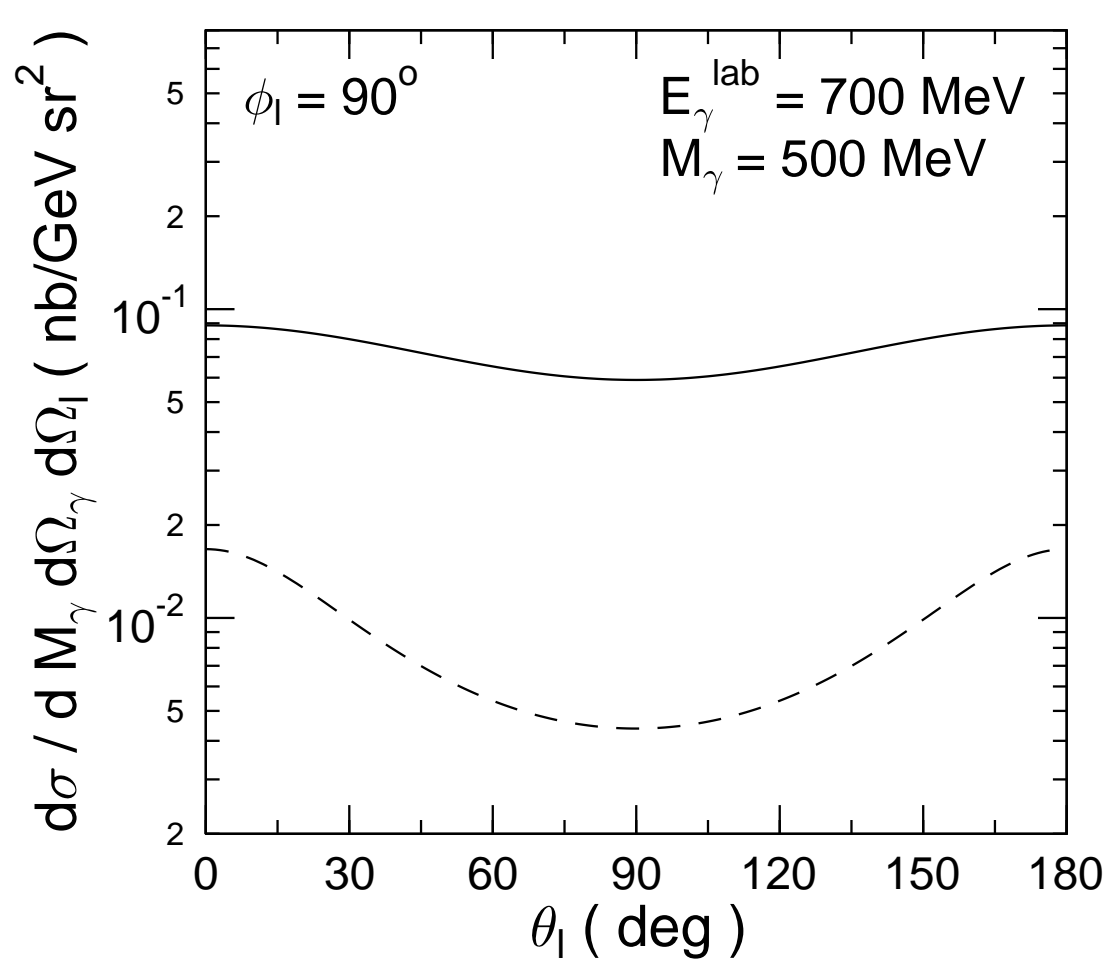

FIG. 4. Exclusive cross section at an incoming energy of $700 \mathrm{MeV}$ in non-coplanar kinematics. Notation for the curves is the same as in Fig. (3). 


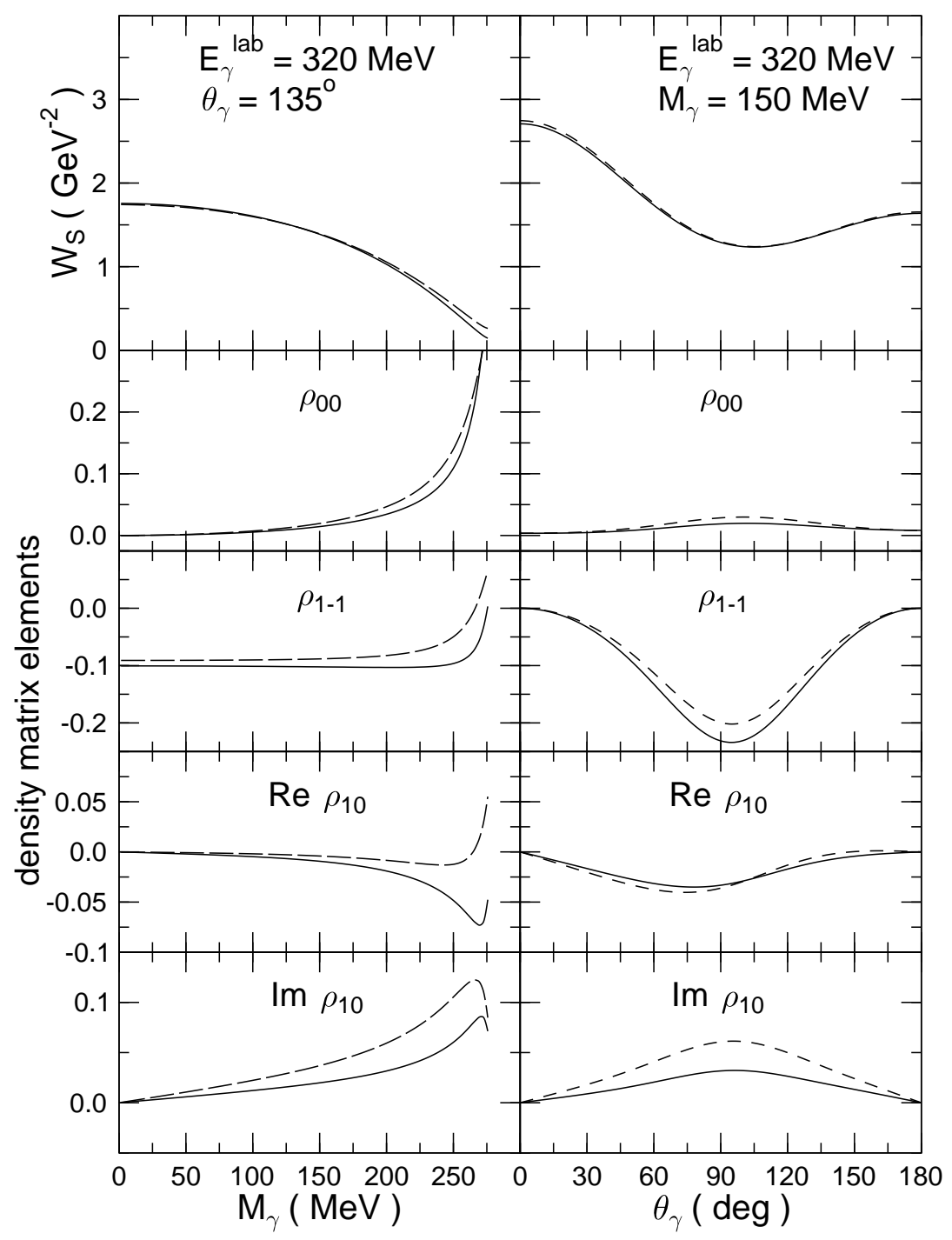

FIG. 5. Response $W_{S}=W_{T}+W_{L}$ (top) and polarization density matrix versus virtual-photon invariant mass $M_{\gamma}$ (left) and photon angle $\theta_{\gamma}$ (right). Incoming photon energy is $320 \mathrm{MeV}$. Solid lines are calculated with the total amplitude, dashed lines without $\sigma$ exchange. 


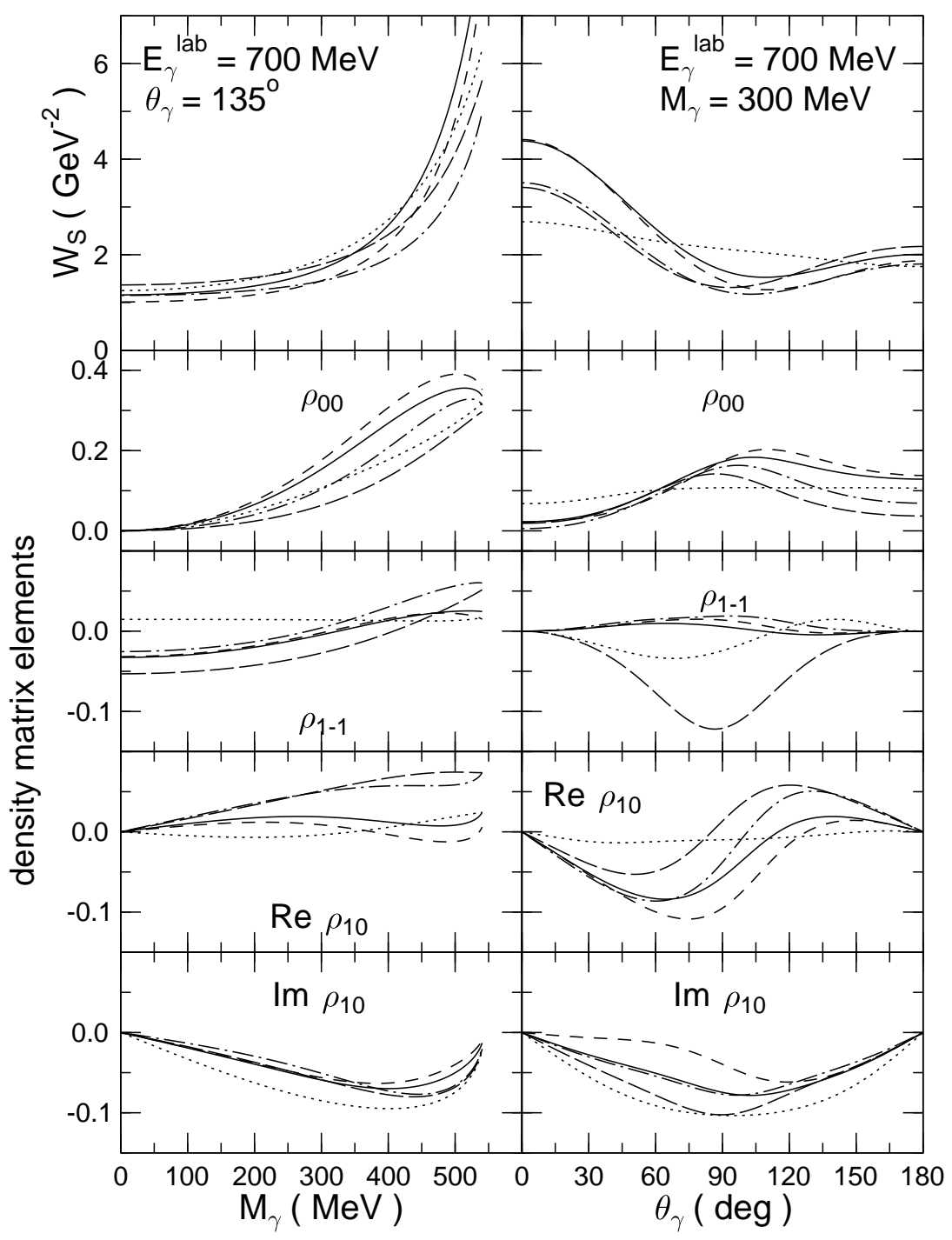

FIG. 6. The same as in Fig. (5) but at photon energy $700 \mathrm{MeV}$. Solid lines show the calculation with the total amplitude (parameter set "B"), dotted lines without $D_{13}(1520)$ resonance, dash-dotted lines without $S_{11}(1535)$ contribution, short-dashed lines without $\sigma$ exchange, and long-dashed lines are calculated with the total amplitude (parameter set "A"). 


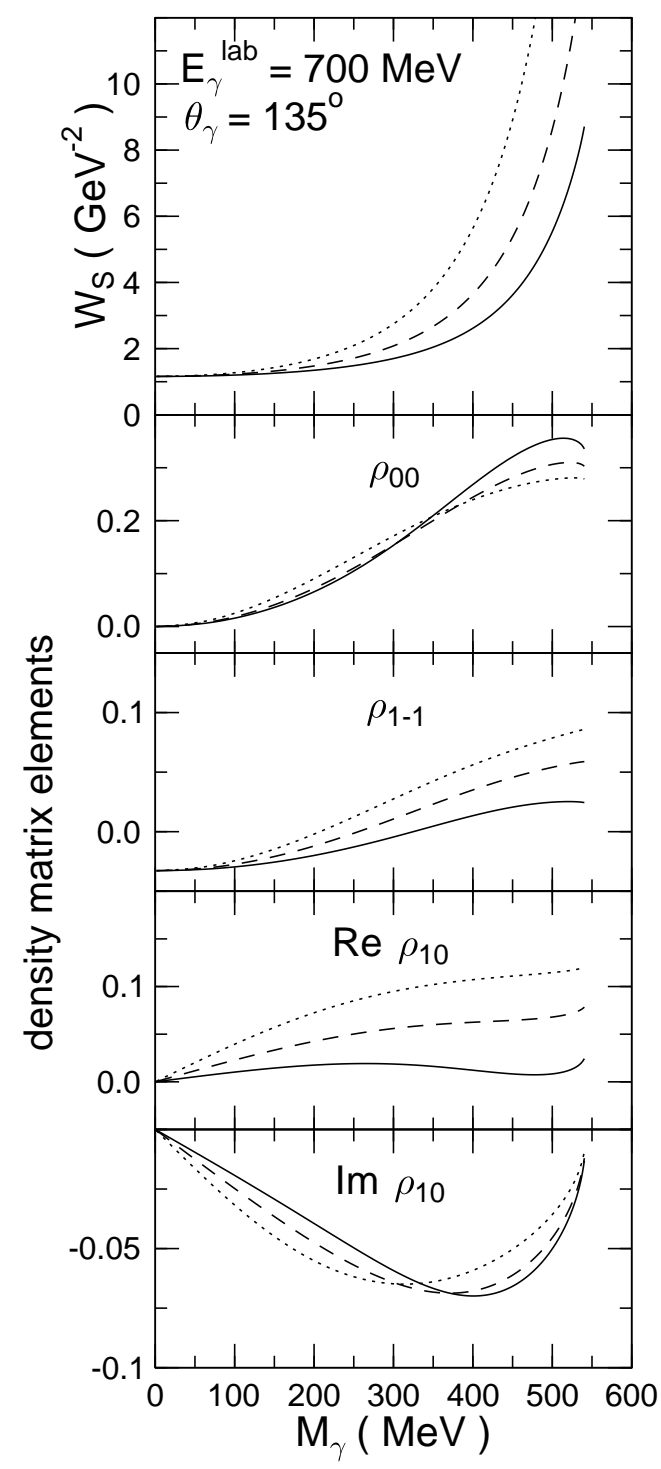

FIG. 7. Response $W_{S}=W_{T}+W_{L}$ (top) and $\rho_{\lambda \lambda^{\prime}}$ calculated for different values of $g_{3}$ in the $\gamma N D_{13}$ vertex Eq. (33). Solid lines: $g_{3}=0$, dashed lines: $g_{3}=-3.3$ (from pion electroproduction), and dotted lines: $g_{3}=-7.9$. Couplings $g_{1}$ and $g_{2}$ are respectively 7.25 and 7.9. 